\title{
The X-ray catalog of spectroscopically identified Galactic $O$ stars
}

\section{Investigating the dependence of X-ray luminosity on stellar and wind parameters ${ }^{\star}$}

\author{
A. Nebot Gómez-Morán ${ }^{1}$ and L. M. Oskinova ${ }^{2,3}$ \\ 1 Observatoire Astronomique de Strasbourg, Université de Strasbourg, CNRS, UMR 7550, 11 rue de l'Université, \\ 67000 Strasbourg, France \\ e-mail: ada.nebot@astro.unistra.fr \\ 2 Institute of Physics and Astronomy, University of Potsdam, 14476 Potsdam, Germany \\ e-mail: lida@astro.physik.uni-potsdam.de \\ 3 Department of Astronomy, Kazan Federal University, Kremlevskaya Str 18, Kazan, Russia
}

Received 18 May 2018 / Accepted 3 August 2018

\begin{abstract}
The X-ray emission of O-type stars was first discovered in the early days of the Einstein satellite. Since then many different surveys have confirmed that the ratio of X-ray to bolometric luminosity in O-type stars is roughly constant, but there is a paucity of studies that account for detailed information on spectral and wind properties of O-stars. Recently a significant sample of O stars within our Galaxy was spectroscopically identified and presented in the Galactic O-Star Spectroscopic Survey (GOSS). At the same time, a large high-fidelity catalog of X-ray sources detected by the XMM-Newton X-ray telescope was released. Here we present the X-ray catalog of $\mathrm{O}$ stars with known spectral types and investigate the dependence of their X-ray properties on spectral type as well as stellar and wind parameters. We find that, among the GOSS sample, $127 \mathrm{O}$-stars have a unique XMM-Newton source counterpart and a Gaia data release 2 (DR2) association. Terminal velocities are known for a subsample of 35 of these stars. We confirm that the X-ray luminosities of dwarf and giant $\mathrm{O}$ stars correlate with their bolometric luminosity. For the subsample of $\mathrm{O}$ stars with measure terminal velocities we find that the $\mathrm{X}$-ray luminosities of dwarf and giant $\mathrm{O}$ stars also correlate with wind parameters. However, we find that these correlations break down for supergiant stars. Moreover, we show that supergiant stars are systematically harder in X-rays compared to giant and dwarf O-type stars. We find that the X-ray luminosity depends on spectral type, but seems to be independent of whether the stars are single or in a binary system. Finally, we show that the distribution of $\log \left(L_{\mathrm{X}} / L_{\mathrm{bol}}\right)$ in our sample stars is non-Gaussian, with the peak of the distribution at $\log \left(L_{\mathrm{X}} / L_{\text {bol }}\right) \approx-6.6$.
\end{abstract}

Key words. stars: massive $-\mathrm{X}$-rays: stars

\section{Introduction}

Since the era of the Einstein X-ray telescope $(0.2-4.0 \mathrm{keV})$ it is known that O-type stars emit X-rays (Harnden et al. 1979). Based on the initial sample of 16 OB stars detected by Einstein, Long \& White (1980) noted that their X-ray luminosity correlates with bolometric luminosity as $L_{\mathrm{X}} \sim 10^{-6 \ldots-8} L_{\mathrm{bol}}$. Pallavicini et al. (1981) extended the study to 35 stars with spectral types in the range from $\mathrm{O} 4$ to $\mathrm{A} 9$ and concluded that the ratio of X-ray to bolometric luminosity is roughly constant ( $L_{\mathrm{X}} \approx(1.4 \pm 0.3) \times 10^{-7} L_{\mathrm{bol}}$ ), breaking down for spectral types later than A5. Later on, Schmitt et al. (1985) showed that the $\log \left(L_{\mathrm{X}} / L_{\mathrm{bol}}\right) \sim-7$ correlation does not hold for A-type stars breaking down already at spectral type B5. Chlebowski et al. (1989) presented "The Einstein X-ray Observatory Catalog of O-type stars". The catalog contains 289 stars with 89 detections and 176 upper limits. It was found that $\mathrm{X}$-ray luminosities of $\mathrm{O}$ stars are in the range $L_{\mathrm{X}} \approx 10^{-5.44 \ldots-7.35} L_{\mathrm{bol}}$.

The ROSAT telescope performed an all-sky X-ray survey (RASS, Voges et al. 1999, 2000) in the $0.2-2.4 \mathrm{keV}$ energy band and detected many OB-type stars, confirming the $L_{\mathrm{X}} \propto L_{\text {bol }}$ corre-

* The X-ray catalog is only available at the CDS via anonymous ftp to cdsarc.u-strasbg. fr (130.79.128.5) or via http://cdsarc. u-strasbg.fr/viz-bin/qcat?J/A+A/620/A89 lation (e.g., Motch et al. 1997). Berghoefer et al. (1997) demonstrated that this correlation flattens considerably below $L_{\mathrm{bol}} \approx$ $10^{38} \mathrm{erg} \mathrm{s}^{-1}$, that is, for mid- and late-type B stars. Since then, the $L_{\mathrm{X}} \propto L_{\mathrm{bol}}$ relation was often revisited and confirmed by many independent studies of OB-stars in clusters and in the field.

With the advent of modern X-ray telescopes XMM-Newton and Chandra, with their broad spectral response $(0.2-12.0 \mathrm{keV})$ and high spatial resolution, the interest in X-ray properties of O stars was once more renewed. Oskinova (2005) verified the $\log \left(L_{\mathrm{X}} / L_{\mathrm{bol}}\right)$ correlation selectively, distinguishing between binary and single stars. A linear regression analysis of a sample of spectroscopic binaries showed a correlation $L_{\mathrm{X}} \approx 10^{-7} L_{\mathrm{bol}}$. It was found that while binary stars are more X-ray luminous than single ones, the correlations between X-ray and bolometric luminosities are similar for both groups. Great effort has been made to study massive star populations in individual clusters (e.g., Moffat et al. 2002; Wolk et al. 2006). Sana et al. (2006) observed the open cluster NGC 6231 using XMM-Newton. Based on a rather small sample of $12 \mathrm{O}$ stars they found a much lower scatter than previous studies $\left(\log \left(L_{\mathrm{X}} / L_{\mathrm{bol}}\right)=-6.91 \pm 0.15\right)$ and showed that the $L_{\mathrm{X}} \propto L_{\mathrm{bol}}$ relation is dominated by soft X-ray emission - the dispersion becomes larger for radiation above $2.5 \mathrm{keV}$.

$\mathrm{X}$-ray emission from a large number of O-stars in the Carina Nebula cluster was studied by Nazé et al. (2011). Using Chandra observations of $60 \mathrm{O}$ stars in this region, a ratio of 
$\log \left(L_{\mathrm{X}} / L_{\mathrm{bol}}\right)=-7.26 \pm 0.21$ was determined. The spectral types were collected from the literature as presented in the contemporaneous Skiff (2014) catalog. Interestingly, using XMM-Newton observations of the same region, Antokhin et al. (2008) determined $\log \left(L_{\mathrm{X}} / L_{\mathrm{bol}}\right)=-6.58 \pm 0.79$. Nazé et al. (2011) explained the discrepancy by the different reddening laws and bolometric luminosities assigned to O-stars in these studies.

An X-ray catalog of OB-stars was presented by Nazé (2009). They cross-correlated the XMM-Newton Serendipitous Source Catalogue: 2XMMi-DR3 (Watson et al. 2009) and the all-sky catalog of Galactic OB stars, which contains 16000 known or "reasonably suspected" OB stars (Reed 2003). Approximately 300 OB stars were found to have an X-ray counterpart. Confirming previous studies, Nazé (2009) showed that X-ray fluxes of O stars are well correlated with their bolometric fluxes. However, the scatter was found to be comparable to that of the RASS studies, that is, much larger than in individual clusters. The average ratio between $\mathrm{X}$-ray (in the $0.5-10.0 \mathrm{keV}$ band) and bolometric fluxes for O-stars was found to be $\log \left(L_{\mathrm{X}} / L_{\mathrm{bol}}\right)=-6.45 \pm 0.51$. Although the Reed (2003) catalog of OB stars is a valuable resource, it is heterogeneous by nature, harvesting data from the SIMBAD data-base $^{1}$, and might contain dubious spectral classifications.

While significant effort firmly established an $L_{\mathrm{X}} \propto L_{\mathrm{bol}}$ correlation for $\mathrm{O}$ stars, its origin is still unknown. A standard explanation of X-ray emission from $\mathrm{O}$ stars is the presence of plasma heated by shocks intrinsic to radiatively driven stellar winds (Lucy \& White 1980; Owocki et al. 1988; Feldmeier et al. 1997). The X-ray luminosity is therefore expected to correlate with stellar wind parameters. One of the most detailed and careful analyses to date was performed by Sciortino et al. (1990), who investigated correlations between X-ray luminosity and stellar and wind parameters based on a large sample of $\mathrm{O}$ stars detected by the Einstein observatory. They found that $L_{\mathrm{X}}$ correlates well with mass, the Eddington factor (that describes relative influence of radiation pressure with respect to gravity), $L_{\mathrm{bol}}$, wind momentum $\left(\dot{M} v_{\infty}\right)$, and kinetic wind luminosity $\left(L_{\text {kin }}\right)$, but only weakly with mass-loss rate $(\dot{M})$ and with terminal wind velocity $\left(v_{\infty}\right)$. Moreover, Sciortino et al. (1990) found that none of these parameters alone can account for the observed dispersion in $L_{\mathrm{X}}$, but that a combination of $L_{\mathrm{bol}}, v_{\infty}$ and $\dot{M}$ is needed. Motivated by the well-known correlation of $L_{X}$ with rotation rate in solar-type stars, Sciortino et al. (1990) searched for similar correlations in hot stars but could not find any evidence.

Alternative and still speculative explanations for X-ray emission from $\mathrm{O}$ stars invoke stellar magnetism. In this case, hot X-ray-emitting plasma could be associated with stellar spots (e.g., Waldron \& Cassinelli 2007). The presence of such magnetic structures in normal stars is gaining support both theoretically and observationally (Cantiello \& Braithwaite 2011; Ramiaramanantsoa et al. 2014).

The aim of the study presented in this paper is to build a homogeneous catalog of X-ray emitting $\mathrm{O}$ stars and to study the dependence of their X-ray emission with stellar and wind properties. The time is now ripe for such a study. The Galactic O-Star Spectroscopic Survey (GOSS) by Sota et al. (2011, 2014) and Maíz Apellániz et al. (2016) is now available. GOSS consists of more than 590 O stars with spectral types homogeneously determined from optical spectroscopy. It is complete down to magnitude $B=8 \mathrm{mag}$. As noted by Sota et al. (2014), the previous studies were subject to discrepancies in spectral type determinations which can lead to errors deriving parameters that depend on spectral types (and propagate to X-ray studies). The X-ray

Wenger et al. (2000). catalog we present in this paper is limited to stars from the GOSS sample for the sake of consistency.

The release of the GOSS coincides with an on-going improvement and expansion of The XMM-Newton Serendipitous Source Catalogue. In this paper we consider GOSS stars with X-ray counterparts in the latest XMM-Newton catalog at the time of writing. We do not include the information from other $\mathrm{X}$-ray surveys and telescopes in order to study the homogeneous $\mathrm{X}$-ray sample representative for the $\mathrm{O}$ star population within our Galaxy. The catalog we present here incorporates distances based on the parallaxes included in the Gaia second data release (DR2).

The paper is structured as follows: in Sect. 2 we describe the $\mathrm{X}$-ray, optical, and infrared associations. Determinations of stellar and X-ray parameters are described in Sects. 3 and 4, respectively. Correlations between X-ray and wind parameters are discussed in Sect. 5, and conclusions are drawn in Sect. 6.

\section{The X-ray, optical, and infrared associations of GOSS and the resulting catalog}

The XMM-Newton satellite has been operating since 2000 (Jansen et al. 2001). There are five X-ray instruments on board: the three European Photon Imaging Cameras (EPIC), and two Reflection Grating Spectrometers (RGS; Strüder et al. 2001; Turner et al. 2001; den Herder et al. 2001). Among the three EPIC cameras, one is equipped with a pn detector and two with Metal Oxide Semi-conductor CCD arrays (MOS cameras). These cover the energy range from 0.2 to $12 \mathrm{keV}$, with an energy resolution $E / \Delta E \approx 50$ at $6.5 \mathrm{keV}$ and with a position accuracy better than $3^{\prime \prime}$ (90\% confidence radius). The catalog we present here is limited to X-ray sources detected by the PN camera.

Thanks to the XMM-Newton large field of view and its high sensitivity, in each pointed observation up to 100 sources are detected serendipitously (Rosen et al. 2016). Since 2003 the XMM-Newton Survey Science Center has been compiling information on serendipitously detected X-ray sources and releasing it to the community in the form of catalogs.

The most recent catalog, released in July 2016, is the 3XMM-DR7; it contains 727790 detections for 499266 unique sources, covering a sky area of about 1032 square degrees. The median flux in the total photon energy band $(0.2-12 \mathrm{keV})$ is $\sim 1.9 \times 10^{-14} \mathrm{erg} \mathrm{cm}^{-2} \mathrm{~s}^{-1}$; in the soft energy band $(0.2-2 \mathrm{keV})$ the median flux is $\sim 6 \times 10^{-15} \mathrm{erg} \mathrm{cm}^{-2} \mathrm{~s}^{-1}$, and in the hard band $(2-12 \mathrm{keV})$ it is $\sim 8 \times 10^{-15} \mathrm{erg} \mathrm{cm}^{-2} \mathrm{~s}^{-1}$. About $23 \%$ of the sources have total fluxes below $1 \times 10^{-14} \mathrm{erg} \mathrm{cm}^{-2} \mathrm{~s}^{-1}$.

To find X-ray counterparts of GOSS stars, their optical positions were cross-matched with the 3XMM-DR7 catalog. We looked for all possible X-ray counterparts within a $3.4 \sigma$ search radius, where $\sigma$ is the combined positional error of the X-ray sources and the optical position of the $\mathrm{O}$ stars: $\sigma=$ $\sqrt{\left(\sigma_{\mathrm{X}}\right)^{2}+\left(\sigma_{\mathrm{opt}}\right)^{2}}$. Here $\sigma_{\mathrm{X}}$ and $\sigma_{\mathrm{opt}}$ are the X-ray and optical positional errors respectively and $\sigma_{\mathrm{opt}}=1^{\prime \prime}$ is assumed.

Thanks to the good angular resolution of XMM-Newton, the source confusion is low - there are only a few cases where two $\mathrm{O}$ stars are unresolved in X-rays (e.g., HD 37742 and HD 37743 both have positions compatible with 3XMM J054045.5-015633), or where a star has two possible $\mathrm{X}$-ray associations and we thus cannot decide which one is the true counterpart (e.g., Cyg OB2-22 A is compatible with both 3XMM J203308.7+411316 and 3XMM J203308.8+411318). We visually inspected these cases, but concluded that $\mathrm{O}$ star and X-ray source cannot be associated unambiguously. After 
Table 1. An example of entry to the GOSXMM catalog and column descriptions.

\begin{tabular}{|c|c|c|c|}
\hline Column Name & & & Explanation \\
\hline GOSS & & 006.01-01.20_01 & Name in the GOSS catalogue \\
\hline NAME & & $9 \overline{\mathrm{Sgr}}$ & Alternative name \\
\hline XMM-NAME & & 3XMM J180352.4-242138 & 3XMM Name as in DR7 \\
\hline GaiaDR2_source_id & & 4066022591147527552 & Gaia source identifier, identical to the Gaia-DR2 source_id. \\
\hline RA & & 180352.446 & RAJ2000 \\
\hline Dec & & -242138.64 & DecJ2000 \\
\hline $\mathrm{M}_{V}$ & $(\mathrm{mag})$ & -5.5 & Absolute magnitude from Martins et al. (2005a) \\
\hline error_M $\mathrm{M}_{V}$ & (mag) & 0.29 & Absolute magnitude error \\
\hline$M_{\mathrm{bol}}$ & (mag) & -9.41 & Bolometric magnitude from Martins et al. (2005a) \\
\hline$e_{-} M_{\mathrm{bol}}$ & (mag) & 0.43 & Bolometric magnitude error \\
\hline$T_{\text {eff }}$ & $(\mathrm{K})$ & 43000 & Effective temperature from Martins et al. (2005a) \\
\hline$e_{-} T_{\mathrm{eff}}$ & $(\mathrm{K})$ & 2000 & Effective temperature error \\
\hline $\log g$ & & 3.92 & Effective gravity from Martins et al. (2005a) \\
\hline$e_{-} \log g$ & & 0.1 & Effective gravity error \\
\hline $\log L_{\mathrm{bol}, \mathrm{S}}$ & $\left(\mathrm{erg} \mathrm{s}^{-1}\right)$ & 5.66 & Bolometric luminosity derived from spectrophotometric distance \\
\hline$v_{\infty}$ & $\left(\mathrm{km} \mathrm{s}^{-1}\right)$ & 2750 & Terminal wind velocity from Prinja et al. (1990) \\
\hline $\log \dot{M}_{\text {Vink }}$ & $\left(M_{\odot} \mathrm{yr}^{-1}\right)$ & -5.62 & Mass-loss rate from the Vink et al. (2000) recipe \\
\hline $\log \dot{M}$ & $\left(M_{\odot} \mathrm{yr}^{-1}\right)$ & -6.32 & Mass-loss rate from this work \\
\hline distance_Gaia & $(\mathrm{kpc})$ & 1.2 & Parallactic distance from Bailer-Jones et al. (2018) \\
\hline min_distance_Gaia & $(\mathrm{kpc})$ & 1.1 & Minimum parallactic distance from Bailer-Jones et al. (2018) \\
\hline max_distance_Gaia & $(\mathrm{kpc})$ & 1.3 & Maximum parallactic distance from Bailer-Jones et al. (2018) \\
\hline distance_ $\mathrm{M}_{V}$ & $(\mathrm{kpc})$ & 1.4 & Spectrophotometric distance \\
\hline error_distance_ $\mathbf{M}_{V}$ & $(\mathrm{kpc})$ & 0.1 & Error on the spectrophotometric distance \\
\hline$B$ & (mag) & 5.94 & $B$ magnitude \\
\hline$e_{-} B$ & (mag) & 0.01 & $B$ magnitude error \\
\hline$V$ & (mag) & 5.96 & $V$ magnitude \\
\hline$e_{-} V$ & (mag) & 0.01 & $V$ magnitude error \\
\hline$E_{B-V}$ & (mag) & 0.26 & Color excess \\
\hline$e_{-} E_{B-V}$ & (mag) & 0.02 & Color excess error \\
\hline$A_{\mathrm{V}}$ & (mag) & 0.81 & Extinction in the $V$ band \\
\hline$e_{-} A_{\mathrm{V}}$ & (mag) & 0.08 & Error in the extinction in the $V$ band \\
\hline $\bar{N}_{\mathrm{H}}$ & $\left(\mathrm{cm}^{-2}\right)$ & $1.5 \mathrm{E} 21$ & Hydrogen column density \\
\hline PN count_rate & $\left(\mathrm{s}^{-1}\right)$ & 1.284 & PN Count rate $(0.2-12 \mathrm{keV})$ \\
\hline error_count_rate & $\left(\mathrm{s}^{-1}\right)$ & 0.017 & PN Count rate error $(0.2-12 \mathrm{keV})$ \\
\hline$f_{\mathrm{X}}$ & $\left(\mathrm{erg} \mathrm{s}^{-1} \mathrm{~cm}^{-2}\right)$ & $4.4 \mathrm{E}-12$ & Unabsorbed X-ray flux $(0.2-12 \mathrm{keV})$ \\
\hline$f_{\mathrm{X}, \min }$ & $\left(\mathrm{erg} \mathrm{s}^{-1} \mathrm{~cm}^{-2}\right)$ & $4.3 \mathrm{E}-12$ & Minimum X-ray flux $(0.2-12 \mathrm{keV})$ \\
\hline$f_{\mathrm{X}, \max }$ & $\left(\mathrm{erg} \mathrm{s}^{-1} \mathrm{~cm}^{-2}\right)$ & $4.4 \mathrm{E}-12$ & Maximum X-ray flux $(0.2-12 \mathrm{keV})$ \\
\hline $\log \left(f_{\text {bol }}\right)$ & $\left(\mathrm{erg} \mathrm{s}^{-1} \mathrm{~cm}^{-2}\right)$ & -5.098 & Bolometric flux \\
\hline$e_{-} \log \left(f_{\mathrm{bol}}\right)$ & $\left(\mathrm{erg} \mathrm{s}^{-1} \mathrm{~cm}^{-2}\right)$ & 0.032 & Bolometric flux error \\
\hline $\log \left(f_{\mathrm{X}} / f_{\mathrm{bol}}\right)$ & & -6.26 & $\mathrm{X}$-ray to bolometric flux ratio \\
\hline $\min \_\log \left(f_{\mathrm{X}} / f_{\text {bol }}\right)$ & & -6.30 & Minimum X-ray to bolometric flux ratio \\
\hline $\max \_\log \left(f_{\mathrm{X}} / f_{\mathrm{bol}}\right)$ & & -6.22 & Maximum X-ray to bolometric flux ratio \\
\hline $\log L_{\mathrm{X}}$ & $\left(\operatorname{erg~s}^{-1}\right)$ & 32.84 & $\mathrm{X}$-ray luminosity $(0.2-12 \mathrm{keV})$ \\
\hline $\log L_{\mathrm{X}, \min }$ & $\left(\operatorname{erg~s}^{-1}\right)$ & 32.74 & minimum X-ray luminosity $(0.2-12 \mathrm{keV})$ \\
\hline $\log L_{\mathrm{X}, \max }$ & $\left(\mathrm{erg} \mathrm{s}^{-1}\right)$ & 32.95 & maximum X-ray luminosity $(0.2-12 \mathrm{keV})$ \\
\hline $\log L_{\mathrm{bol}, \mathrm{P}}$ & $\left(\mathrm{erg} \mathrm{s}^{-1}\right)$ & 39.10 & Bolometric luminosity derived from parallactic distance \\
\hline $\min \_\log L_{\mathrm{bol}, \mathrm{P}}$ & $\left(\mathrm{erg} \mathrm{s}^{-1}\right)$ & 38.98 & Minimum bolometric luminosity \\
\hline max_log $L_{\mathrm{bol}, \mathrm{P}}$ & $\left(\mathrm{erg} \mathrm{s}^{-1}\right)$ & 39.24 & Maximum bolometric luminosity \\
\hline HR2 & & -0.493 & Hardness ratio HR2 \\
\hline error_HR2 & & 0.013 & Error on the hardness ratio HR2 \\
\hline HR3 & & -0.814 & Hardness ratio HR3 \\
\hline error_HR3 & & 0.017 & Error on the hardness ratio HR3 \\
\hline
\end{tabular}

discarding these objects, our final sample contains 135 stars from the GOSS uniquely associated with a 3XMM-DR7 X-ray source.

As a next step we collected the optical and infrared photometry for our sample stars. Our sample catalog was crossmatched with the GSC2.3.2 and the 2MASS Point Source Catalogs (2MASS-PSC). The former is an all sky catalog based on the all-sky photographic surveys from the Palomar and UK Schmidt telescopes (DSS), while the latter is an all sky survey covering approximately the 4 to 16 magnitude range in three bands ( $J, H$ and $K_{\mathrm{s}}$; Cutri et al. 2003). Except for HD 93128, all our sample stars have counterparts within $1^{\prime \prime}$ in the GSC2.3.2 and the 2MASS-PSC catalogs. 

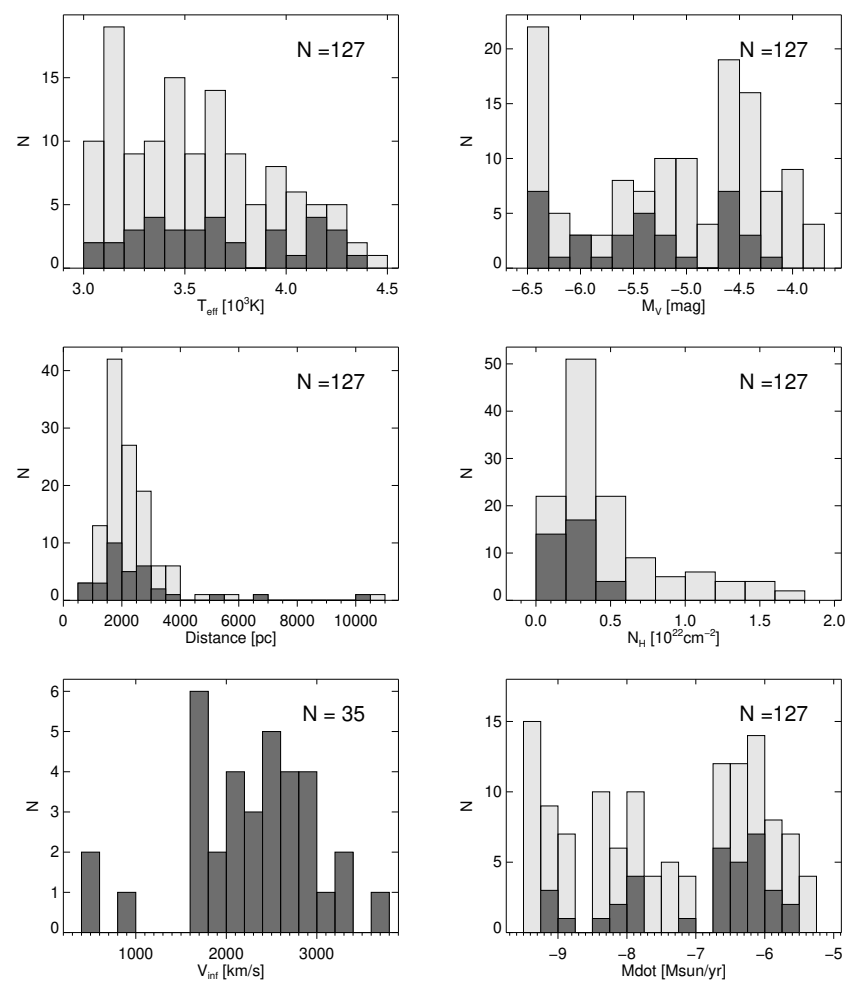

Fig. 1. Histograms showing distributions of stellar parameters for Ostars in our catalog. From left to right and from top to bottom: effective temperatures, absolute magnitudes, parallactic distances, neutral hydrogen column densities, stellar wind velocities, and mass-loss rates for the whole sample (127 O stars shown in light gray), and for stars with known terminal velocities (35 stars shown in dark gray).

We exclude HD 93128 from the follow up study since it is located in a crowded field and its photometry is not reliable, as well as the known high-mass X-ray binaries HD 153919 and LM Vel (Liu et al. 2006; Martínez-Núñez et al. 2017). This reduces our sample to $132 \mathrm{O}$ stars detected with the XMMNewton PN camera and uniquely identified.

The full sample of our stars is presented in a table that is accessible in electronic form via the VizieR database ${ }^{2}$. Table 1 shows an example of the parameters provided for each star in our catalog, which includes distances, X-ray, and stellar properties.

\section{Stellar parameters}

All our sample stars have well determined spectral types. This provides the basis for assigning fundamental parameters to each object. To estimate stellar wind parameters we use an empiric approach. We collect stellar wind parameters from the most recent literature for a sub-sample of our objects, and extrapolate them to the rest of our sample stars. Figure 1 shows the distribution of the effective temperatures, absolute magnitudes, distances, neutral hydrogen column densities, stellar wind velocities, and mass-loss rates for our sample stars.

\subsection{Fundamental stellar parameters}

Stellar effective temperatures, $\log (g)$, masses, radii and absolute magnitudes are assigned to each sample star according to its spectral type using the calibrations from Martins et al.

\footnotetext{
2 Ochsenbein et al. (2000).
}

(2005a). Since in this work the tabulated values are limited to luminosity classes V, III, and I, the values for luminosity classes IV and II were interpolated. For luminosity classes $\mathrm{Ia}, \mathrm{Ib}$, and Iab, we assumed absolute magnitudes and effective temperatures of supergiant stars. The peak in the absolute magnitude distribution seen in Fig. 1 at about -6.4 mag corresponds to $\mathrm{O}$ supergiant stars. To estimate errors on stellar parameters we assumed an uncertainty on the spectral subtype of \pm 1 , we then determined the corresponding stellar parameters and fixed errors to the maximum difference between these determined stellar parameters; for example, $\sigma_{T_{\text {eff, }, \mathrm{SpT}}}=\max \left(\left|T_{\mathrm{eff}, \mathrm{SpT}}-T_{\text {eff, }, \mathrm{SpT}-1}\right|,\left|T_{\mathrm{eff}, \mathrm{SpT}}-T_{\mathrm{eff}, \mathrm{SpT}+1}\right|\right)$. For estimating $\sigma_{\log (g)}$ we performed a similar analysis but assuming an uncertainty on the luminosity class of one subclass.

Among the sample of $\mathrm{O}$ stars studied here, 38 sources are known to be spectroscopic binaries (Sota et al. 2014). For those cases we display the spectral type of the primary stars. Three sources are known to be magnetic: $\theta^{1}$ Ori, HD 57682, and CPD 592629 (Donati et al. 2002; Grunhut et al. 2009; Nazé et al. 2014).

\subsection{Extinction}

Extinction was calculated using color excesses $E_{B-V}, E_{J-K_{\mathrm{s}}}$, and $E_{H-K_{\mathrm{s}}}$ in the optical and in the infrared. The intrinsic colors are from Martins \& Plez (2006). In the optical we calculated the extinction using the relation $A_{\mathrm{V}}=R_{\mathrm{V}} \times E_{B-V}$ with $R_{\mathrm{V}}=3.1$. In the infrared we used the extinction relations $\mathrm{A}_{K}=0.67 \times E_{J-K_{\mathrm{s}}}$ and $\mathrm{A}_{K}=1.82 \times E_{H-K_{\mathrm{s}}}$ from Indebetouw et al. (2005). Then, the extinction relation $\mathrm{A}_{K}=0.114 \times A_{\mathrm{V}}$ (Cardelli et al. 1989) provides an alternative way to estimate $A_{\mathrm{V}}$.

For a set of $O$ stars in common with Jenkins (2009) we compared the color excess $E_{B-V}$ and found that, in general, the values we derive here agree well with Jenkins (2009). We also compared the optical excesses obtained from the infrared colors. Although we find a good general agreement, the scatter is larger than when using optical colors only. Therefore to estimate the extinction for our program stars we use $E_{B-V}$ as derived from optical photometry.

There are various relations in the literature that link the neutral hydrogen column density to the color excess, which range from $N_{\mathrm{H}} \sim 1 \times 10^{21} \times E_{B-V} \mathrm{~cm}^{-2}$ to $N_{\mathrm{H}} \sim 9 \times 10^{21} \times E_{B-V} \mathrm{~cm}^{-2}$ (Predehl \& Schmitt 1995; Gudennavar et al. 2012; Liszt 2014). In this work we adopt $N_{\mathrm{H}}=5.6 \times 10^{21} \times E_{B-V} \mathrm{~cm}^{-2}$ (Bohlin et al. 1978). In Sect. 4.1 we consider in detail how this choice affects the estimates of X-ray fluxes of our sample stars.

For two objects ( $v$ Ori and $15 \mathrm{Mon}$ A) the estimated $N_{\mathrm{H}}$ is very low. We set $N_{\mathrm{H}}$ for both stars equal to a minimum value of $10^{18} \mathrm{~cm}^{-2}$.

\subsection{Distance}

The distances to our sample stars were estimated by using both the spectrophotometric and the parallactic methods.

We started by estimating distances from the absolute visual magnitudes derived using bolometric corrections and the photometry of our program stars. The $V$ magnitudes were corrected for the extinction (see Sect. 3.2) and the errors on the spectrophotometric distances were derived by propagating the errors on magnitudes and extinction.

As a next step, we searched the extended Gaia DR2 cata$\log$ (Bailer-Jones et al. 2018), and found that the parallactic distances are estimated for 127 of our sample stars. These were compared with the spectrophotometric distances we derived. 
Reassuringly, the spectrophotometric distances agree well with the parallactic ones. Therefore, in the following we use the Gaia DR2 parallactic distances.

While most of the GOSS sources are within $4 \mathrm{kpc}$, six sources are estimated to be at larger distances: CPD -59 2600 (estimated distance $d=4.9 \mathrm{kpc}$ ) is a member of the open cluster $\mathrm{Cl}$ Trumpler 16 for which distances in the literature range from 2 to $5.4 \mathrm{kpc}$ (Mel'Nik \& Dambis 2009; Pandey et al. 2010). HD $168076 \mathrm{AB}$ (estimated distance $d=5.1 \mathrm{kpc}$ ), is a member of the open cluster M 16 at a distance of $1.7 \mathrm{kpc}$ (Kharchenko et al. 2005). HD 159176 (estimated distance $d=$ $6.6 \mathrm{kpc}$ ) and HD 93403 (estimated distance $d=10.2 \mathrm{kpc}$ ) are found at distances of 1 and $2.4 \mathrm{kpc}$, respectively, according to Megier et al. (2009). Finally, for LS 4067 AB (estimated distance $d=10.7 \mathrm{kpc}$ ) and ALS 18769 (estimated distance $d=$ $5.5 \mathrm{kpc}$ ), we found no distances in the literature.

\subsection{Terminal wind velocities and mass-loss rates}

The O star winds are radiatively driven (Castor et al. 1975). While grasping the fundamental physics, currently the stellar wind theory has limited predictive power. At present, it is not yet possible to theoretically calibrate stellar wind parameters in dependence on spectral types. In this situation, different approaches to estimating wind parameters for a large sample of $\mathrm{O}$ stars are possible. First, a full multi-wavelength spectroscopic analysis of each star in a sample could yield their stellar and wind properties. This method is impractical due to, for example, observational challenges. Besides, any stellar atmosphere model used for spectral analysis has its own limitations. Second, the fundamental stellar parameters could be used as input for theoretical recipes predicting wind velocities and mass-loss rates. This is the easiest and therefore most commonly used option convenient routines are publicly available (Vink et al. 2000). In Table 1 we list the theoretical mass-loss rates for each star in our sample ( $\left.\log \dot{M}_{\text {Vink }}\right)$. However, there are notable discrepancies between the empirically measured mass-loss rates and those predicted by the theoretical recipes (e.g., Fullerton et al. 2006). Especially dramatic is the situation for late O-dwarfs, where the models fail (Muijres et al. 2012). Such stars constitute a significant part of our sample. Third, a semi-empirical approach that builds on existing measurements is a practical way to estimate wind parameters for a large number of stars. From UV spectroscopy, stellar wind velocities are securely measured for 35 stars of our sample. Furthermore, recent spectroscopic analyses using sophisticated stellar atmosphere models were performed for 25 stars in our sample (see Table 2). Importantly, these stars belong to different spectral types and were analyzed with different independent stellar atmosphere models. Hence, the number of empirically well studied stars is sufficiently large to establish an empirical calibration of wind properties that show a dependency on spectral type. We choose this option to study the dependence of X-ray luminosity on stellar wind parameters.

Using UV spectra of resonance lines obtained by the International Ultraviolet Explorer, Prinja et al. (1990) measured the terminal velocity in a large number of OB-type star winds ${ }^{3}$; among them 35 belong to our sample. Their wind velocities are typically above $\sim 1000 \mathrm{~km} \mathrm{~s}^{-1}$. Winds of two stars, HD $93521\left(v_{\infty} \sim 490 \mathrm{~km} \mathrm{~s}^{-1}\right)$ and $\theta^{1}$ Ori $\left(v_{\infty} \sim 580 \mathrm{~km} \mathrm{~s}^{-1}\right)$, are unusually slow. Another object with a comparably slow wind is HD $152408\left(v_{\infty}=955 \mathrm{~km} \mathrm{~s}^{-1}\right)$. Interestingly, while $\theta^{1}$ Or is a

\footnotetext{
3 We note that the errors on the velocity measurements are not provided by Prinja et al. (1990).
}

Table 2. Stellar mass-loss rates of O type stars collected from the literature.

\begin{tabular}{lccc}
\hline \hline HD Name & SpT & $\dot{M}\left(M_{\odot} \mathrm{yr}^{-1}\right)$ & Ref. \\
\hline \multicolumn{4}{c}{ Luminosity class I } \\
HD 66811 & O4I & $2.5 \mathrm{e}-6$ \\
HD 16691 & O4I & $3.0 \mathrm{e}-6$ & 1 \\
HD 190429A & O4If & $2.1 \mathrm{e}-6$ & 2 \\
HD 15570 & O4I & $2.8 \mathrm{e}-6$ & 1 \\
HD 14947 & O4.5If & $2.8 \mathrm{e}-6$ & 1 \\
HD 210839 & O6.5I & $1.6 \mathrm{e}-6$ & 1 \\
HD 163758 & O6.5I & $1.6 \mathrm{e}-6$ & 2 \\
HD 192639 & O7.5I & $1.3 \mathrm{e}-6$ & 1 \\
\hline \multicolumn{4}{c}{ Luminosity class II-III } \\
\hline HD 93250 & O4III & $5.6 \mathrm{e}-7$ \\
HD 15558 & O4.5III & $1.9 \mathrm{e}-6$ & 3 \\
HD 190864 & O6.5III & $4.6 \mathrm{e}-7$ & 4 \\
HD 34656 & O7II & $3.0 \mathrm{e}-7$ & 4 \\
HD 24912 & O7.5III & $4.0 \mathrm{e}-7$ & 4 \\
HD 36861 & O8III & $3.0 \mathrm{e}-7$ & 4 \\
$\delta$ Ori Aa1 & O9.5II & $4.0 \mathrm{e}-7$ \\
\hline \multicolumn{4}{c}{5} \\
\hline HD 46223 & Luminosity class V \\
HD 15629 & O4V & $3.2 \mathrm{e}-7$ \\
O4.5V 93204 & O5.5V & $3.2 \mathrm{e}-7$ & 3 \\
HD 42088 & O6V((f) z & $1.8 \mathrm{e}-7$ & 3 \\
HD 152590 & O7.5Vz & $1.6 \mathrm{e}-8$ & 3 \\
HD 93028 & O9IV & $1.0 \mathrm{e}-9$ & 3 \\
HD 46202 & O9.2V & $1.3 \mathrm{e}-9$ & 3 \\
HD 38666 & O9.5V & $3.0 \mathrm{e}-10$ & 3 \\
HD 34078 & O9.5V & $3.0 \mathrm{e}-10$ & 3 \\
HD 36512 & O9.7V & $5.0 \mathrm{e}-10$ & 6 \\
\hline
\end{tabular}

Notes. An interested reader is strongly encouraged to consult original papers for details of the spectroscopic analyses.

References. (1) Šurlan et al. (2013); (2) Bouret et al. (2012); (3) Martins et al. (2005a); (4) Repolust et al. (2004), note that the mass-loss rates in the table are reduced by a facor of 3; (5) Shenar et al. (2015); (6) Shenar et al. (2017).

well-known magnetic star, the search for magnetic fields in HD 93521 and HD 152408 did not reveal any (Grunhut et al. 2017; Schöller et al. 2017).

As illustrated in Fig. 2, terminal velocity decreases with stellar spectral type since stars with lower effective temperatures tend to have lower escape velocities (Prinja et al. 1990). While the stellar wind theory predicts a relationship between the terminal velocity and the photospheric escape velocity (Abbott 1978; Lamers \& Cassinelli 1999), the scatter seen in Fig. 2 implies that the situation in real objects may be more complicated, further justifying our empirical approach.

Although only 35 stars have measured terminal velocities, they cover the whole range of possible stellar parameters (see Fig. 1), and therefore can be taken as representative of the whole sample.

While stellar wind velocities could be directly measured from UV spectra, measuring mass-loss rates typically requires the model fitting of spectral lines. The errors on the measured mass-loss rates are largely systematic, reflecting the limitations of the applied models. Presently, the most sophisticated stellar atmosphere models do not rely on the assumption of local-thermodynamical equilibrium (LTE; i.e., are non-LTE), include line-blanketing, and account for wind clumping (e.g., 


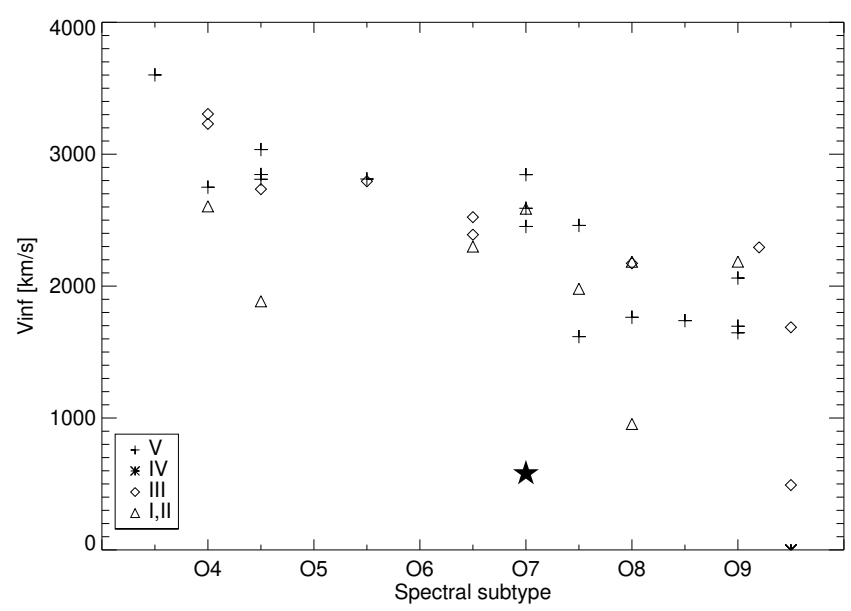

Fig. 2. Terminal wind velocities (from Prinja et al. 1990) as a function of the spectral type for $\mathrm{O}$ stars of different luminosity classes (see legend, the asterisk denotes a known magnetic star).

Hillier \& Miller 1998; Hamann \& Gräfener 2003; Puls et al. 2005).

We collected literature where the mass-loss rates of our sample stars are obtained from spectroscopic analyses done with advanced model atmospheres. To the best of our knowledge, we found such analyses for $25 \mathrm{O}$ stars in our sample. Each of these models has its own way of describing the effects of wind clumping. The mass-loss rates are compiled in Table 2 and are the face values from the corresponding papers (except for luminosity class II-III; see discussion below).

Among the main problems affecting empirical measurements of mass-loss rates in O stars is stellar wind clumping. Significant effort has been dedicated to evaluating the effects of wind inhomogeneity on spectral analysis and to improve model stellar atmospheres (see e.g., Oskinova et al. 2016). The current understanding is that the in case of $\mathrm{O}$ supergiants, the theoretical recipes (Vink et al. 2000) predict mass-loss rates that are $\sim 1.5-3$ times higher than those obtained from spectroscopic analyses that include the effects of optically thin ("microclumping") as well as optically thick clumping ("macroclumping"; e.g., Oskinova et al. 2007; Sundqvist et al. 2011; Šurlan et al. 2012; Shenar et al. 2015).

Bouret et al. (2012) presented measurements of wind parameters in a sample of eight Galactic $\mathrm{O}$ supergiants. They neglected macroclumping (i.e., it was explicitly assumed that all clumps are optically thin). In order to achieve consistent fits of lines in the optical and UV, quite small clump volume filling factors were adopted (of about 0.05 ; see the original paper for exact definitions and details of the spectral analysis). On the other hand, Šurlan et al. (2013) used the 3D Monte-Carlo radiative transfer model to measure mass-loss rates for five Galactic $\mathrm{O}$ supergiants. The mass-loss rates they derived are systematically higher by a factor of two compared to those of Bouret et al. (2012). Šurlan et al. (2013) emphasized that an adequate treatment of the line formation in inhomogeneous winds is a prerequisite for interpreting $\mathrm{O}$-star spectra and determining mass-loss rates. They showed that mass-loss rates of O-type supergiants derived using macroclumping techniques are lower by a factor of 1.3 2.6 than the mass-loss obtained from the theoretical recipes by Vink et al. (2000). Therefore, comparing the results obtained by different models with the results of the theoretical recipes, we conclude that the spectroscopically derived mass-loss rates of O-type supergiants are accurate within a factor of approximately three.

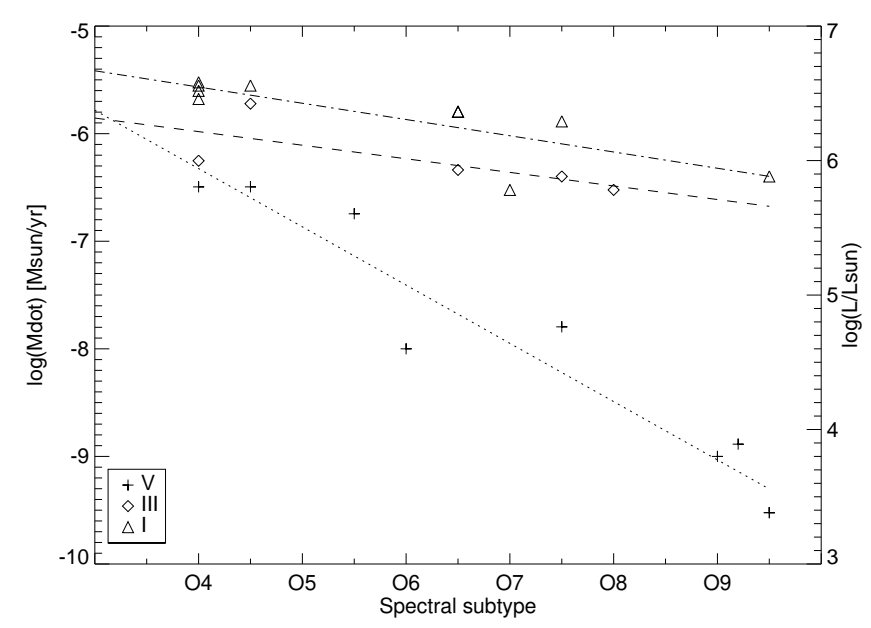

Fig. 3. Empirically derived mass-loss rates as a function of the spectral type for $\mathrm{O}$ stars of luminosity classes I, III, and V (see legend). Lines show linear fits for each luminosity class. The mass-loss rates of giants and supergiants are uncertain within a factor of $2-3$, i.e., $\Delta \log \dot{M} \approx$ $0.3-0.5$.

To our knowledge, only a few Galactic O-type giants have been analyzed by advanced clumped stellar atmosphere models (Martins et al. 2005b; Shenar et al. 2015). On the other hand, a significant number of O-type giants were included in the study of Repolust et al. (2004), who measured mass-loss rates using optical spectra and presented the results without correcting for clumping effects. As they note, these values tend to overpredict the real mass-loss rates. To correct the mass-loss rates in giant stars, we note that Oskinova et al. (2007) have shown that the emission in $\mathrm{H} \alpha$ line is not affected by macroclumping even in the dense winds of $\mathrm{O}$ supergiants, and therefore can be used as a good mass-loss rate indicator. However, the derived mass-loss rates still have to be corrected because of optically thin clumping. Adopting a conservative value of 0.1 for the volume filling factor, the O-giant mass-loss rates from Repolust et al. (2004) are reduced by a factor of three. The resulting mass-loss rates are given in Table 2 and Fig. 3.

To illustrate the uncertainties associated with the mass-loss measurements and justify our approach, it is useful to consider an example: the star HD 93250 is classified as O3V((f)) in Repolust et al. (2004), who obtained its mass-loss rate $\log \dot{M}=$ $-5.46_{-0.16}^{+0.11}$. Assuming a filling factor of 0.1 , the mass-loss rate should be reduced to $\log \dot{M}=-5.9$. Martins et al. (2005b) classify HD 93250 as $\mathrm{O} 3.5 \mathrm{~V}((\mathrm{f}+))$. Using clumped models with a filling factor of 0.01 , they measure its mass-loss rate as $\log \dot{M}=$ $-6.25 \pm 0.7$. On the other hand, the theoretical mass-loss rate for this star is an order of magnitude higher, $\log \dot{M}_{\text {Vink }}=-5.25$. In GOSS, the star is re-classified as O4III. Correspondingly, the theoretical mass-loss rate is $\log \dot{M}_{\text {Vink }}=-5.42$. This example illustrates the large discrepancy between theoretical and empiric mass-loss rates, but it shows that the empirical measurements agree within a factor of two to three when clumping is taken into account. This example also illustrates that the errors are largely dominated by the systematic differences between the models. Furthermore, each atmosphere model has errors on mass-loss rates that are due to the errors on the fundamental stellar parameters, distance, and the adopted description of clumping.

The mass-loss rates of O-type dwarfs are much less certain compared to giants and supergiants. Still, the empirically derived mass-loss rates of low-luminosity $\mathrm{O}$ dwarfs are orders of mag- 
nitude lower than predicted by theoretical recipes. This problem is often referred to as "the weak wind problem" (Martins et al. $2005 \mathrm{~b}$ ). It is interesting to note that nearly all solutions suggested to resolve the weak-wind problem in $\mathrm{O}$ stars invoke X-rays (Huenemoerder et al. 2012, and references therein). The empirically measured mass-loss rates of well-studied Galactic O-dwarfs are given in Table 2. The strong dependence of their mass-loss rate on the spectral type is clearly seen in Fig. 3 which shows empiric mass-loss rates as a function of spectral type for each luminosity class. It is interesting to note that the dependence of mass-loss rate on spectral subtype is much more pronounced in dwarf stars than in supergiants.

We performed a linear fit to the recent empiric $\dot{M}$ estimates (as described above) and obtained the following scaling relations,

$\log \left(\dot{M}\left[M_{\odot} \mathrm{yr}^{-1}\right]\right)=-4.2-0.5 \times \mathrm{SpT}$ for OV stars,

$\log \left(\dot{M}\left[M_{\odot} \mathrm{yr}^{-1}\right]\right)=-5.5-0.1 \times \mathrm{SpT}$ for OIII stars,

$\log \left(\dot{M}\left[M_{\odot} \mathrm{yr}^{-1}\right]\right)=-5.0-0.2 \times \mathrm{SpT}$ for OI stars,

where "SpT" denotes a corresponding numeric value of a spectral subtype; for example, for an O4I star, the value of SpT is 4.

Keeping in mind the significant uncertainties in mass-loss measurements, these empiric relations were used to assign the mass-loss rates to the $\mathrm{O}$ stars in this study.

\section{X-ray parameters}

\subsection{X-ray fluxes}

The 3XMM-DR7 catalog provides count rates in the $0.2-12 \mathrm{keV}$ energy band for each source. To calculate X-ray fluxes on this basis, one needs to establish the energy count rate to flux conversion factor (ECF). For this purpose we used the Portable, Interactive Multi-Mission Simulator (PIMMS). The intrinsic X-ray spectrum emitted by an O-type star was approximated by a collisional plasma model, APEC (Smith et al. 2001), with solar abundances and single temperature $k T=0.35 \mathrm{keV}$, which is typical for single X-ray emitting O stars (Nazé et al. 2011). For each source in our sample, we calculated unabsorbed X-ray fluxes from the mean PN count rate of all detections. The upper and lower values for the X-ray fluxes based on the mean errors of the count rates were used to estimate the errors of the fluxes. The fluxes were corrected for interstellar absorption using the $N_{\mathrm{H}}$ values calculated in Sect. 3.2. All the X-ray observations were carried out with medium or thick filters meaning that optical loading should not be an issue. Nevertheless, as a sanity check, we investigated the summary source detection flag SC_SUM_FLAG and confirmed that no sources in our catalog were flagged for suspected optical loading. As a following step, $\mathrm{X}$-ray luminosities were computed for the distances calculated as described in Sect. 3.3. All stars in our sample have X-ray luminosities in the range $10^{31-34} \mathrm{erg} \mathrm{s}^{-1}$, for either spectrophotometric or parallactic distance estimates.

Among our sample stars, 60 objects have been observed by XMM-Newton only once. The rest of the sources have been observed and detected 3-4 times on average, and up to 11 times, with individual exposure times ranging from 3 to $100 \mathrm{ks}$. The unabsorbed fluxes were calculated using the average value of the count rates among all detections for each source. Therefore the fluxes and luminosities presented in this catalog characterize steady X-ray emission of O stars.
O-type stars show typical X-ray variability on the level of 10\% (e.g., Oskinova et al. 2001; Nazé et al. 2013; Massa et al. 2014). Time series were automatically extracted by the $X M M$ Newton pipeline for 108 out of the 132 O stars we study here. Seven of the stars are classified in the 3XMM-DR7 catalog as variable based on a $\chi^{2}$ variability test: $\theta^{1}$ Ori C, HD 193322A, HD 152218, $\sigma$ Ori AB, HD 97434, $\zeta$ Pup, and HD 93129A. An analysis of their X-ray variability is beyond the scope of this paper.

Besides count rates, the 3XMM-DR7 catalog also provides $\mathrm{X}$-ray fluxes for all objects. Comparing them with the fluxes we derived for our sample stars, we find a mean difference in the flux ratio between the XMM-Newton catalog and our derived values of $\approx 1.65$. This difference is due to 1 ) the different adopted $\mathrm{X}$-ray spectral models; the $3 \mathrm{XMM-DR7}$ fluxes are calculated under assumption of a power-law type X-ray spectrum, while we use a thermal plasma model; and 2) the more accurate interstellar neutral hydrogen densities towards $\mathrm{O}$ stars which we derive in this study.

To place an upper limit on the X-ray luminosity of an O-star which was observed but not detected by XMM-Newton, we built the cumulative distribution function of X-ray fluxes of all sources detected within the same field of view as listed in 3XMM-DR7 catalog. The upper flux limit to a non-detected O star could then be calculated as the flux correction factor 1.65 multiplied by the flux at which $90 \%$ of other field sources are detected.

We searched for non-detections and placed upper limits on their X-ray flux. Firstly we searched for O stars within $900^{\prime \prime}$ of the center of any XMM-Newton observation, that is, within the field of view. We ensured that the optical positions were within $\mathrm{X}$-ray images, that is, not falling into gaps or outside of X-ray image boundaries (especially for observations performed not in the full window model). Nine O-type stars were observed but are not detected by XMM-Newton.

$\mathrm{O}$ stars not detected in X-rays have late spectral subtype in the range O6-O9.5, cover all luminosity classes and have upper $\mathrm{X}$-ray to bolometric flux ratio close to -7 . Since the number of non-detected stars is small, we do not expect the distribution of $\log \left(L_{\mathrm{X}} / L_{\text {bol }}\right)$ (see Sect. 4.2) to be biased towards high X-ray to bolometric flux ratio.

The choice of the plasma temperature and $N_{\mathrm{H}}$ affects the calculation of the ECFs and, consequently, the estimated X-ray fluxes. To investigate how robust our derived X-ray luminosities are, we calculated the ECFs for two different plasma temperatures, $k T=0.35 \mathrm{keV}$ and $k T=0.5 \mathrm{keV}$, and for a wide range of $N_{\mathrm{H}}$ values. As illustrated in Fig. 4, a different choice of the plasma temperature in a plausible range leads to an only slightly different ECF. On the other hand, the ECF's dependence on the $N_{\mathrm{H}}$ increases for larger $N_{\mathrm{H}}$ values. The average $N_{\mathrm{H}}$ of our sample stars is $7 \times 10^{21} \mathrm{~cm}^{-2}$ (see Fig. 1). As can be seen from Fig. 4, changing this value by a factor 1.5 results in variation of the ECF by a factor of two. Hence, the ca lculated X-ray fluxes depend mainly on the adopted values of $N_{\mathrm{H}}$.

There are 34 stars in common between ours and Nazé (2009) catalogs. The fluxes we obtained for these stars are a factor of two higher, chiefly because we adopted a different relation between $E_{B-V}$ and $N_{\mathrm{H}}$ in our study.

\subsection{Correlation between $X$-ray and bolometric luminosity of O-type stars}

Considering the relation between X-ray and bolometric fluxes, $\log \left(f_{\mathrm{X}} / f_{\text {bol }}\right)$, allows us to ignore the problems associated 


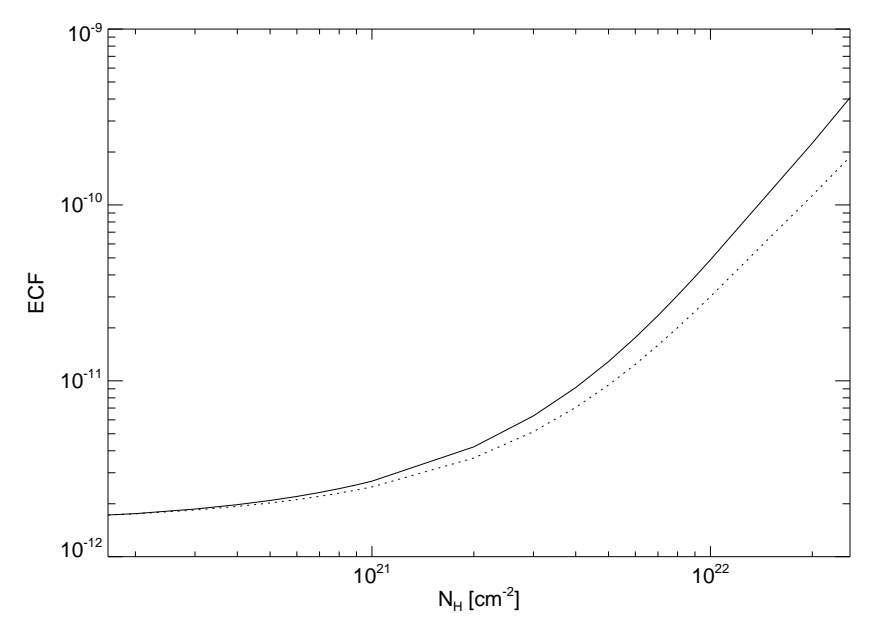

Fig. 4. ECF calculated assuming an APEC model with solar abundance and temperature of $0.35 \mathrm{keV}$ (solid line) and for $0.5 \mathrm{keV}$ (dotted line) as a function of $N_{\mathrm{H}}$.

with the uncertainties in distances. The bolometric fluxes are calculated according to spectral types of our sample stars as

$\log \left(f_{\text {bol }}\right)=-4.6-\left(V-A_{\mathrm{V}}+\mathrm{BC}_{\mathrm{V}}\right) / 2.5$.

Figure 5 displays the $\log \left(f_{\mathrm{X}} / f_{\mathrm{bol}}\right)$ relation for the full sample, independent on their luminosity class. Confirming previous studies, we find that the X-ray fluxes scale with the bolometric fluxes. The linear regression fit yields the correlation

$\log \left(f_{\mathrm{X}}\right)=-6.21+1.05 \times \log \left(f_{\mathrm{bol}}\right)$

This result is obtained for the total energy band of the $X M M$ Newton PN camera $(0.2-12 \mathrm{keV})$. For completeness we include in Appendix A a similar analysis carried out for two different energy bands (a soft and a hard one).

The bolometric luminosities, $L_{\mathrm{bol}}$, were derived in two ways. The distance-independent bolometric luminosities are based on spectral type (Martins et al. 2005a). The distance-dependent luminosities $\left(L_{\mathrm{bol}}=4 \pi d^{2} f_{\text {bol }}\right)$ are based on bolometric fluxes as obtained using Eq. (5) and Gaia DR2 distances (these luminosities are included in Table 1). There is good agreement between bolometric luminosities derived by different methods. We use the distance dependent luminosity since it eliminates distance dependence in $\log \left(L_{\mathrm{X}} / L_{\mathrm{bol}}\right)$.

Figure 6 shows the $\log \left(L_{\mathrm{X}} / L_{\mathrm{bol}}\right)$ distribution for all our sample stars. The $\log \left(L_{\mathrm{X}} / L_{\mathrm{bol}}\right)$ distribution is non-Gaussian, with a peak at around -6.7 and minimum and maximum values at around -7.5 and -5.0 respectively, corroborating previous results based on ROSAT data (Berghoefer et al. 1997). The impact of errors is discussed in Appendix B. As can be seen from Fig. 6 the distribution shows a tail towards high values. Binarity could result in an increased X-ray flux, for example, due to wind-wind collisions. In the following sections we discuss the dependence of $\log \left(L_{\mathrm{X}} / L_{\mathrm{bol}}\right)$ on luminosity class, spectral type, and binarity.

Although the $\log \left(L_{\mathrm{X}} / L_{\mathrm{bol}}\right)$ distribution is non-Gaussian, we computed average values for $\log \left(f_{\mathrm{X}} / f_{\text {bol }}\right)$ for all stars as a function of the spectral type, for single and binary stars and for two different ranges of spectral types. The results are presented in Table 4.

\subsubsection{The dependence of $\log \left(L_{X} / L_{\text {bol }}\right)$ on luminosity class}

The $\log \left(f_{\mathrm{X}} / f_{\text {bol }}\right)$ relation for different luminosity classes is shown in Fig. 7. The correlation coefficients between X-ray and bolometric fluxes and the $1-\sigma$ confidence intervals of the coefficients of the linear fits are given in Table 3. To measure the strength of the linear correlation, we calculated the Pearson correlation coefficient $R$. This coefficient can take values between 1 and -1 . Values close to 1 or -1 reflect a strong positive or negative linear correlation between the two variables, while values close to 0 indicate no relationship.

Dwarfs and giant $\mathrm{O}$ stars show a very strong correlation between $\log \left(f_{\mathrm{X}}\right)$ and $\log \left(f_{\text {bol }}\right)$. The O6 IV star ALS 15108 has unusually high $\log \left(f_{\mathrm{X}}\right)$ and $\log \left(f_{\mathrm{bol}}\right)$. We investigated this star in more detail, and found that it has a very high reddening $\left(E_{B-V} \sim 3.1\right)$. The star belongs to the Cygnus OB2 association. The extinction is known to be large in the area. It is likely that we overestimate the neutral hydrogen column density towards this star, leading to its unusually high X-ray luminosity.

The correlation between the X-ray and the bolometric for $\mathrm{O}$ supergiants is only moderate ${ }^{4}$. Despite the relatively small number of O-supergiants in our study, one can see that confirmed spectroscopic binary supergiants have approximately constant bolometric flux $\left(\log \left(f_{\text {bol }}\right) \sim-5.5\right)$, while their X-ray fluxes span over three orders of magnitude (Fig. 7).

The $\log \left(L_{\mathrm{X}} / L_{\mathrm{bol}}\right)$ distribution depends on the luminosity class, as illustrated in Fig. 8. For dwarf and giant stars the distribution peaks at around -6.6 , shifting towards smaller values, -6.8 , for supergiant stars. Mean values are $-6.6 \pm 0.4,-6.5 \pm 0.4$, and $-6.4 \pm 0.6$ for dwarf, giant, and supergiant stars, respectively. Interestingly, all these averages are above the canonical value of -7 . It is important to keep in mind that the $\log \left(L_{\mathrm{X}} / L_{\mathrm{bol}}\right)$ distribution is non-Gaussian.

To study the dependence of the $\log \left(L_{\mathrm{X}} / L_{\mathrm{bol}}\right)$ distribution on spectral type, we divided our sample into two groups - early $\mathrm{O}$ stars with spectral subtypes in the range $\mathrm{O} 2-\mathrm{O} 6$, and late $\mathrm{O}$ stars with spectral subtypes from O6 to O9.5. The $\log \left(L_{X} / L_{\text {bol }}\right)$ distribution peaks around -6.4 for early O-type stars, and shifts towards lower values close to -6.6 for later spectral types. The mean values are $-6.3 \pm 0.5$ and $-6.6 \pm 0.4$ for early and late spectral types, respectively.

\subsection{2. $\log \left(L_{x} / L_{\text {bol }}\right)$ dependence on binarity}

The distribution of $\log \left(L_{\mathrm{X}} / L_{\mathrm{bol}}\right)$ for confirmed binaries is very similar to that of single stars (see Fig. 10). Both distributions peak at $\log \left(L_{\mathrm{X}} / L_{\mathrm{bol}}\right) \sim-6.6$ and the mean values are $-6.6 \pm 0.5$ and $-6.4 \pm 0.5$ for single and binary stars, respectively. This corroborates the results of previous studies (Sciortino et al. 1990; Oskinova 2005; Nazé 2009). Most likely, the true fraction of binaries in our sample is significantly higher than those of the already confirmed binaries, and the list of punitively single stars is very likely contaminated by binaries. A thorough analysis of the confirmed single star sample and confirmed colliding wind binaries is beyond the scope of this paper.

\subsection{X-ray hardness-ratios}

Among other parameters, the 3XMM-DR7 catalog provides hardness ratios (HR) describing the difference in count rates

4 Interestingly, the weak or absent correlation between X-ray and bolometric luminosity was previously noticed in another class of massive stars with dense and fast winds, namely Wolf-Rayet stars (Ignace et al. 2000). 


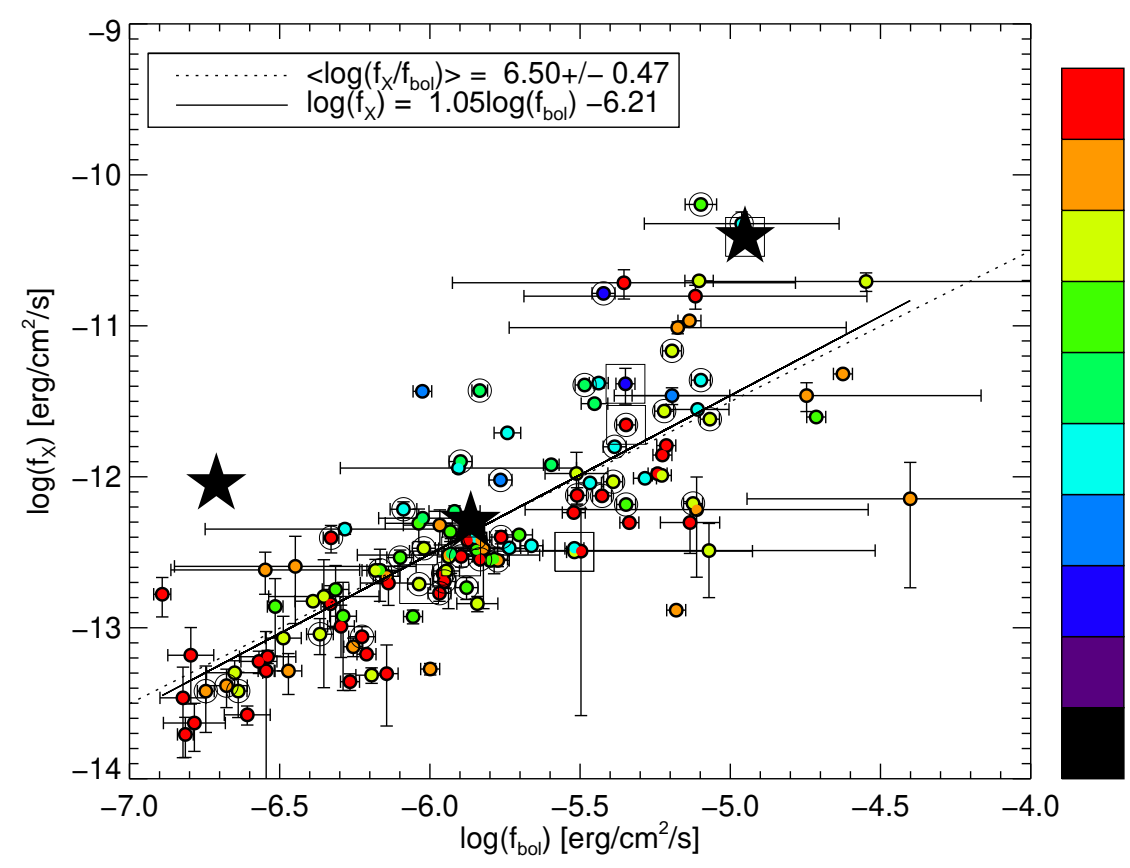

4 Fig. 5. X-ray flux vs. bolometric flux for $\mathrm{O}$ 3 stars of all luminosity classes and as a function 3 of the spectral subtype (color-coded with ear2 lier subtypes being bluer, and the later subtypes 2 being redder). Known spectroscopic binaries are highlighted with a ring around the solid circle, magnetic $\mathrm{O}$ stars are represented by asterisks, and stars flagged as variable are shown with a squared symbol. The linear fit $\log \left(f_{\mathrm{X}}\right)=$ $a+b \times \log \left(f_{\text {bol }}\right)$ and the mean $\log \left(f_{\mathrm{X}} / f_{\text {bol }}\right)$ value are shown as solid and dotted lines, respectively (see text for details).

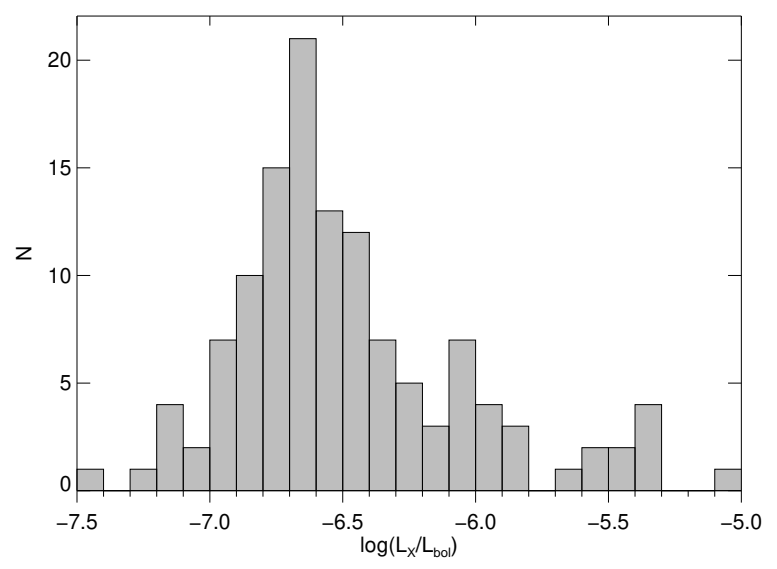

Fig. 6. Distribution of the X-ray to bolometric luminosity ratio for all the stars in the sample.

Table 3. Number of $\mathrm{O}$ stars, correlation coefficient $R$ (see text for its definition), and fit coefficients for $\log \left(f_{\mathrm{X}}\right)=a+b \times \log \left(f_{\mathrm{bol}}\right)$.

\begin{tabular}{ccccc}
\hline \hline Lum. Class & $\#$ & $R$ & $a$ & $b$ \\
\hline All & 127 & 0.78 & $-6.21 \pm 0.44$ & $1.05 \pm 0.08$ \\
V & 60 & 0.80 & $-7.05 \pm 0.54$ & $0.92 \pm 0.09$ \\
IV & 14 & 0.83 & $-7.92 \pm 0.94$ & $0.78 \pm 0.16$ \\
V+IV & 74 & 0.80 & $-7.15 \pm 0.47$ & $0.90 \pm 0.08$ \\
III & 21 & 0.89 & $-3.91 \pm 1.03$ & $1.45 \pm 0.18$ \\
I+II & 30 & 0.55 & $-6.19 \pm 1.63$ & $1.04 \pm 0.30$ \\
\hline
\end{tabular}

between two consecutive bands, defined as

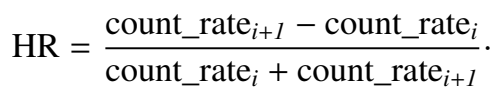

In other words, the HR gives a rough idea of the slope of the $\mathrm{X}$-ray spectrum; for example, $\mathrm{HR}=0$ would be a flat spectrum, HR $>0$ implies the source emits more photons in the harder band, and $\mathrm{HR}<0$ tells us the source emits more photons in the
Table 4. Mean $\log \left(f_{\mathrm{X}} / f_{\text {bol }}\right)$.

\begin{tabular}{cc}
\hline \hline Lum. Class & $\log \left(f_{\mathrm{X}} / f_{\text {bol }}\right)$ \\
\hline All & $-6.50 \pm 0.47$ \\
V & $-6.56 \pm 0.39$ \\
IV & $-6.61 \pm 0.25$ \\
V+IV & $-6.57 \pm 0.37$ \\
III & $-6.51 \pm 0.42$ \\
I+II & $-6.40 \pm 0.62$ \\
SpT early & $-6.23 \pm 0.44$ \\
SpT late & $-6.62 \pm 0.43$ \\
Single & $-6.54 \pm 0.47$ \\
Binaries & $-6.41 \pm 0.46$ \\
\hline
\end{tabular}

softer band. The HR value 1 or -1 in 3 XMM-DR7 means the source was not detected in either band.

We calculate the mean PN hardness ratios HR2 and HR3 that describe emission in the energy bands 2 and 3 , and 3 and 4 , and cover the energy ranges $0.5-1.0,1.0-2.0$, and $2.0-4.5 \mathrm{keV}$, respectively.

Figure 11 shows the relation between HRs and $N_{\mathrm{H}}$ limited to sources with errors on HRs lower than 0.3. One can see that, as expected, HR2 increases with $N_{\mathrm{H}}$. There are only a few sources with high HR2 and HR3 at a relatively low $N_{\mathrm{H}}$, these are intrinsically hard X-ray sources. We computed mean HR in bins of similar $N_{\mathrm{H}}$ for different luminosity classes, and found that supergiant stars have higher HRs at the same $N_{\mathrm{H}}$, as can be seen in particular in the HR2 (right upper panel of Fig. 11) but also in the HR3. This shows that supergiant stars have systematically harder spectra than dwarfs.

\section{Correlations between X-ray and wind parameters}

In this section we investigate the correlations between $\mathrm{X}$ ray luminosity and stellar wind parameters for different spectral types and luminosity classes. As explained in detail in Sect. 3.4, the stellar wind velocities were measured from the UV 

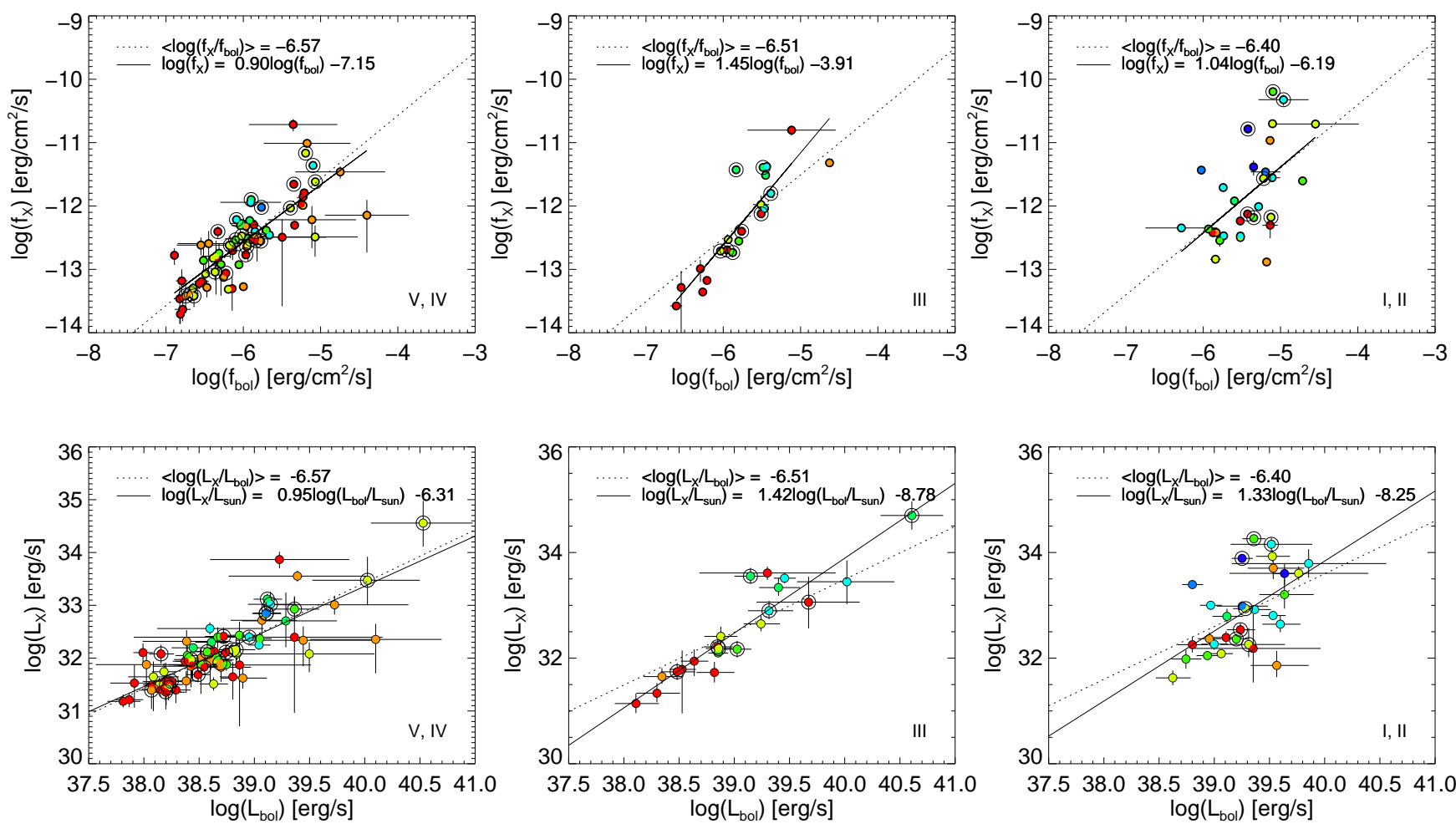

Fig. 7. $\log \left(f_{\mathrm{X}} / f_{\text {bol }}\right)$ (top row) and $\log \left(L_{\mathrm{X}} / L_{\mathrm{bol}}\right)$ (bottom row) relation for $\mathrm{O}$ stars of different luminosity classes (V, III, and I from left to right panels). Known spectroscopic binaries are highlighted with a ring around the solid circle, and spectral types are color coded as in Fig. 5. The linear fits $\log \left(f_{\mathrm{X}}\right)=a+b \times \log \left(f_{\text {bol }}\right)$ and the mean $\log \left(f_{\mathrm{X}} / f_{\text {bol }}\right)$ values for each case are shown as solid and dotted lines, respectively (see text for details). For $\mathrm{O}$ supergiants, the correlations are weak, while they are significant for $\mathrm{O}$ dwarf and giant stars (see Table 3 ).

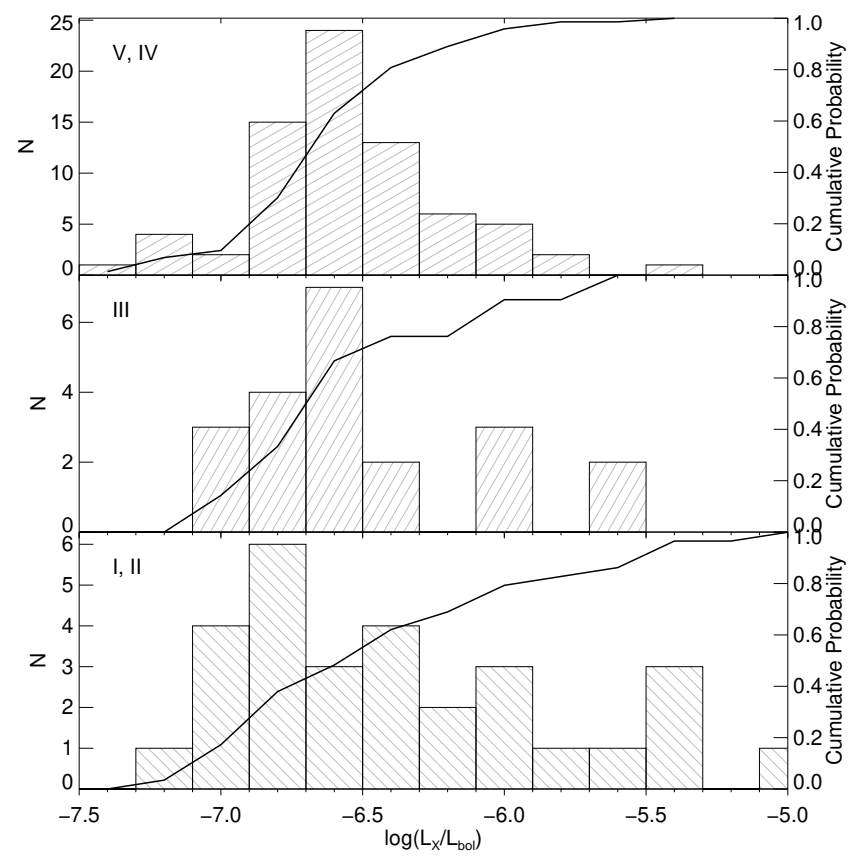

Fig. 8. $\log \left(L_{\mathrm{X}} / L_{\mathrm{bol}}\right)$ histogram and cumulative distribution function for dwarfs (top panel), giants (middle panel), and supergiants (bottom panel).

spectra only for 35 stars in our sample. The wind velocities show a general trend of decreasing for later spectral types (see Fig. 2), however with a significant scatter. The scatter is also obvious in Fig. 3 which shows the dependence of mass-loss rates on

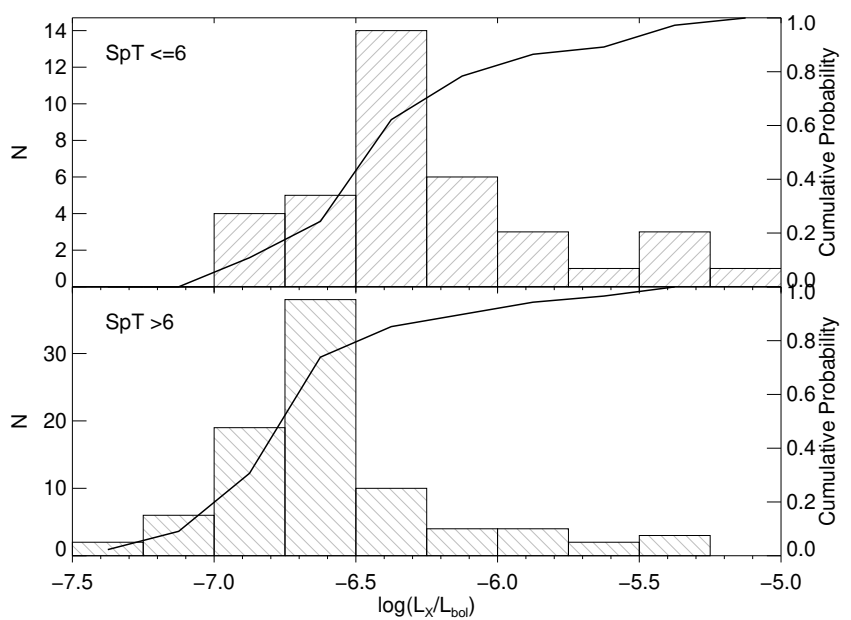

Fig. 9. $\log \left(L_{\mathrm{X}} / L_{\text {bol }}\right)$ histogram and cumulative distribution function for early O2-O6 subtypes (top panel) and late O6-O9.5 subtypes (bottom panel).

spectral subtype for stars of different luminosity classes. The uncertainty on empirically derived mass-loss rates is about a factor of three. The errors on wind velocities and mass-loss rates propagate to other wind parameters, such as wind kinetic energy and momentum.

In Fig. 12 the correlation between X-ray luminosity and wind parameters is shown. To reduce the errors, only the stars with wind velocities empirically measured from the UV spectra are included in this figure. Hence the upper row in Fig. 12 displays only the measured quantities of the X-ray luminosities and wind velocities for a sub-sample of $\mathrm{O}$ stars. 


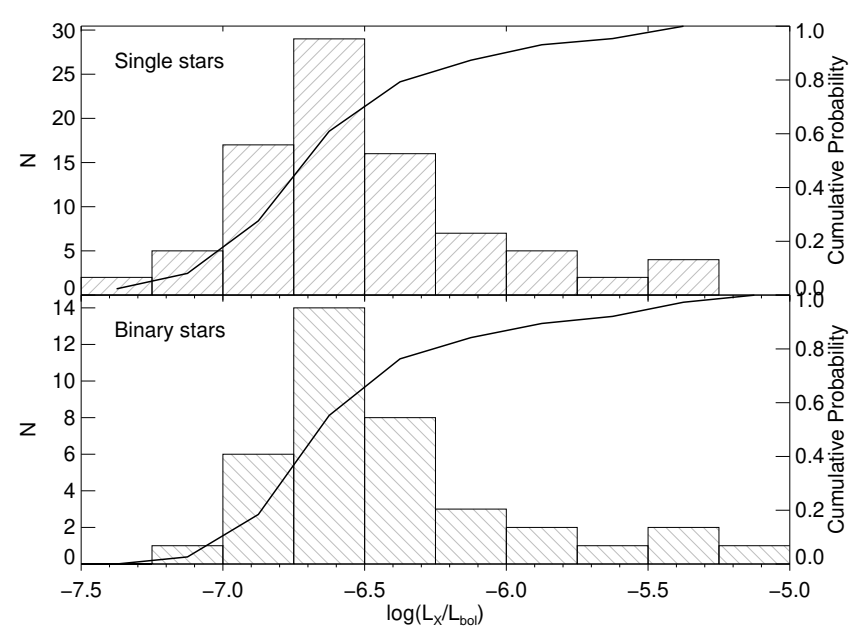

Fig. 10. $\log \left(L_{\mathrm{X}} / L_{\mathrm{bol}}\right)$ histogram and cumulative distribution function for putative single stars (top panel), and in binaries (bottom panel).

The linear fits were performed without taking into account the magnetic stars, and also omitting HD 93521, given its unusually slow wind. We note that there are three other peculiar systems with rather low $L_{\mathrm{X}}$ and low stellar wind density: HD 14633 (ON8.5V), BN Gem (O8:V, the lowest X-ray luminosity among the whole sample stars), and HD 15137 (O9.5IIIII). Results of the linear fits are given in Table 5; the errors in the coefficients correspond to the $1-\sigma$ confidence interval.

The correlation between X-ray luminosity and wind velocity is seen for dwarf and giant stars, but is absent for supergiants. As can be seen in Fig. 12, for dwarf and giant stars the X-ray luminosities increase as winds increase in speed. Despite relatively large scatter (in particular for dwarfs) which is largely induced by binaries with their higher X-ray luminosities, the overall correlation is weak for dwarfs and strong for giant stars. For single stars this correlation implies that stars with later spectral types, that is, stars with lower effective temperatures and lower $v_{\infty}$, have lower X-ray luminosities. The situation is different for supergiants, where only very weak or no correlation between X-ray luminosity and terminal velocity is found. This may reflect the reality or be simply due to the small number of supergiants with measured $v_{\infty}$.

Since the wind velocities and X-ray luminosities are empiric measurements, the detected correlations (or their absence) are physical and not induced by systematic errors. This gives us confidence to search for correlations between X-ray luminosities and other wind parameters.

Since spectral types are known for all stars in our sample we can estimate their mass-loss rates using the SpT- $\dot{M}$ scaling relations Eqs. (1)-(3) derived in Sect. 3.4. As can be seen from Fig. 12 and Table 5, the X-ray luminosities show weak correlation with mass-loss rate for main sequence and giant stars, while there is basically no correlation for supergiants.

The correlation is also present when considering spectral types within each luminosity class - later spectral subtypes tend to have lower mass-loss rates and lower X-ray luminosities than early spectral subtypes.

As a next step we investigate the scaling of X-ray luminosity with global parameters describing stellar winds, such as the wind kinetic luminosity $L_{\text {kin }}=\frac{1}{2} \dot{M} \times v_{\infty}{ }^{2}\left[\mathrm{erg} \mathrm{s}^{-1}\right]$, the density estimator $N_{1}=\frac{\dot{M}}{v_{\infty}}\left[\mathrm{g} \mathrm{cm}^{-1}\right]$, and the modified stellar wind momentum,
$D=\dot{M} \times v_{\infty} \times \sqrt{\frac{R_{*}}{R_{\odot}}}$, defined according to Kudritzki \& Puls (2000). Reflecting the scaling of X-ray luminosity with mass-loss rate and wind velocity, the giant $\mathrm{O}$ stars show strong correlations with wind parameters, while dwarfs show only weak correlations and no correlation is seen in $\mathrm{O}$ supergiants.

The X-ray luminosity of late O-type dwarfs (exhibiting the weak wind problem; see Sect. 3.4) is a few percent of the stellar wind kinetic luminosity (see Fig. 12). This fraction drops for earlier spectral subtypes and for giants and supergiants; for example, in the latter case $L_{\mathrm{X}}<10^{-3} L_{\text {kin }}$. The trend showing larger $\mathrm{X}$-ray output from $\mathrm{O}$ stars with weaker winds has already been reported (e.g., Oskinova et al. 2006).

The density parameter $N_{1}$ is a proxy for the mean wind density, and determines the shock cooling length (see Eq. (14) and its discussion in Hillier et al. 1993). Owocki et al. (2013) noticed that for radiative shocks one can expect that $L_{\mathrm{X}} \propto\left(\dot{M} / v_{\infty}\right)^{1-m}$. Using the theoretically expected scaling between mass-loss rates and bolometric luminosities, they suggested that if the exponent $m \approx 0.4$ the observed scaling between $L_{\mathrm{X}}$ and $L_{\mathrm{bol}}$ can be reproduced.

We test this suggestion using our empiric approach. As can be seen from Fig. 12 and Table 5, for the total ensemble of $\mathrm{O}$ stars, the correlation between $\log \left(L_{\mathrm{X}}\right)$ and $\log N_{\mathrm{l}}$ is very weak. Only giants show a significant correlation which is different from the one expected theoretically.

Considering the modified wind momentum, we notice similar trends as for other wind parameters - a significant correlation is observed for dwarfs and giants, while no correlation is seen for supergiant stars in Fig. 12. The analysis by Mokiem et al. (2007) confirmed theoretical expectations that modified wind momenta do not depend on stellar luminosity class (when not accounting for the weak wind star problem). In this study we choose the empiric approach, and for low-luminosity dwarfs we adopt empiric mass-loss rates which are significantly lower that those predicted theoretically. Figure 12 shows that the scaling relations between X-ray luminosity and wind parameters are quite different for different luminosity classes.

To measure the strength of the correlation between X-ray luminosity and wind parameters we calculated the Pearson correlation coefficients. We also used a non-parametric method, in particular we calculated the Spearman's rank correlation coefficient $\rho$. The Spearman coefficient can take values between 1 and -1 . Values close to 1 or -1 reflect a strong positive or negative monotonic correlation (not necessarily a linear correlation such as for the Pearson coefficient) between the two variables, while values close to 0 indicate no relationship. Results are listed in Table 5. Values obtained for $R$ and $\rho$ suggest that the X-ray luminosity of supergiant stars is not correlated with wind parameters. We provide linear fits between X-ray luminosity and wind parameters for each luminosity class in Table 5. For supergiant stars, fitting the $\mathrm{X}$-ray luminosity to a constant gives $\log \left(L_{X}\right)=32.68 \pm 0.21$.

This raises the key question on the origin of the $L_{\mathrm{X}}-L_{\mathrm{bol}}$ correction for O-stars - is the X-ray luminosity determined by fundamental stellar parameters or by wind strength? - a problem analogous to the question "which came first, the chicken or the egg?". In the former case, X-rays could be associated with smallscale magnetic fields on the stellar photosphere (Oskinova et al. 2011, and references therein), which in turn depend on subsurface structure (Cantiello \& Braithwaite 2011; Urpin 2017). The latter case is the well accepted scenario of X-ray generation by strong shocks in stellar winds resulting from the line driven instability (Feldmeier et al. 1997). 

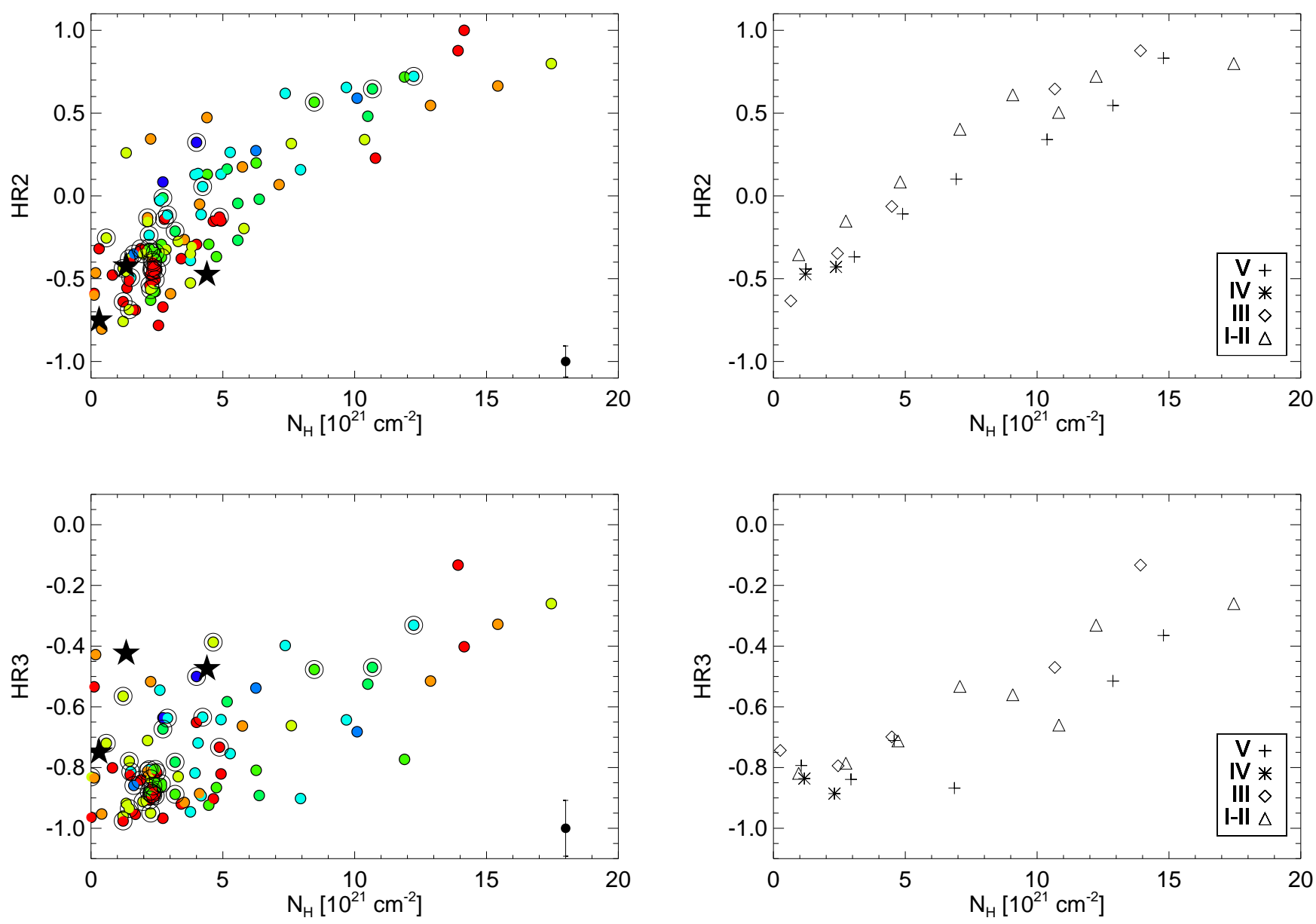

Fig. 11. Hardness ratios HR2 (top panels) and HR3 (bottom panels) as a function of $N_{\mathrm{H}}$. In the left panels symbols have been colored according to the spectral type as in Fig. 5 and mean error in the HR is indicated in the lower right corner of the plot. In the right panels, mean HRs have been calculated per bin of $N_{\mathrm{H}}$ and different luminosity classes; different symbols stand for different luminosity classes as indicated in the legend.

As mentioned earlier, these results are based on a limited sample of 35 stars with measured stellar wind velocities. Although these wind velocities are decreasing towards later spectral subtypes, there is a significant scatter at each spectral subtype, a scatter also present in the dependence of mass-loss rates on spectral subtype for stars of different luminosity classes. Errors on wind velocities and mass-loss rates propagate to other wind parameters, such as wind kinetic energy and momentum. A larger sample of stars with measured wind velocities and mass-loss rates would help to better constrain the correlations between X-ray luminosity and stellar wind parameters.

For completeness, in Appendix $\mathrm{C}$ we show the correlations between bolometric luminosity and stellar wind parameters.

\section{Summary and conclusions}

The spectroscopic sample of Galactic O stars, GOSS (Sota et al. 2014; Maíz Apellániz et al. 2016), was used to investigate, in a homogeneous and consistent way, the X-ray emission from $\mathrm{O}$ stars. We found that 127 stars from GOSS have an unambiguous counterpart in the 3XMM-DR7 catalog of X-ray sources and are associated to a Gaia-DR2 source. The stellar parameters of $\mathrm{O}$ stars were obtained using spectral calibrations presented by Martins et al. (2005a). Mass-loss rates of O stars of different spectral types and luminosity classes were compiled from the literature and used to derive an empirical spectral-type mass-loss rate relation. From this empirical relation we derived mass-loss rates for all our sample stars. Terminal velocities from Prinja et al. (1990) are available for a subsample of 35 of these stars. For this subsample of $\mathrm{O}$ stars, wind parameters (kinetic luminosity, momentum and density) were estimated based on the empirically derived mass-loss rates and the measured terminal velocities.

Interstellar extinction was estimated on the basis of optical photometry available for all our sample stars. The stellar distances were estimated on the basis of stellar photometry as well using Gaia DR2. The X-ray hardness ratios and fluxes were calculated from count rates provided in the 3XMM-DR7 catalog, while X-ray luminosities are based on dereddened fluxes and Gaia DR2 parallactic distances.

We confirm that X-ray luminosities of dwarf and giant $\mathrm{O}$ stars correlate with their bolometric luminosities. For the subsample of $\mathrm{O}$ dwarf and giant stars with known terminal velocities we find that their X-ray luminosities are correlated with their key wind parameters, such as terminal velocity, mass-loss rate, wind kinetic energy, and wind momentum. However, these correlations break down for supergiant stars. Furthermore, O supergiants have systematically harder X-ray spectra than dwarf and giant stars at any given interstellar extinction. We show that the distribution of $\log \left(L_{\mathrm{X}} / L_{\mathrm{bol}}\right)$ in our sample is non-Gaussian, with the peak of the distribution at $\log \left(L_{\mathrm{X}} / L_{\mathrm{bol}}\right) \approx-6.6$, minimum value $\log \left(L_{\mathrm{X}} / L_{\text {bol }}\right) \approx-7.5$, and a longer tail towards higher 
A. Nebot Gómez-Morán and L. M. Oskinova: X-ray catalog of spectroscopically identified Galactic O stars
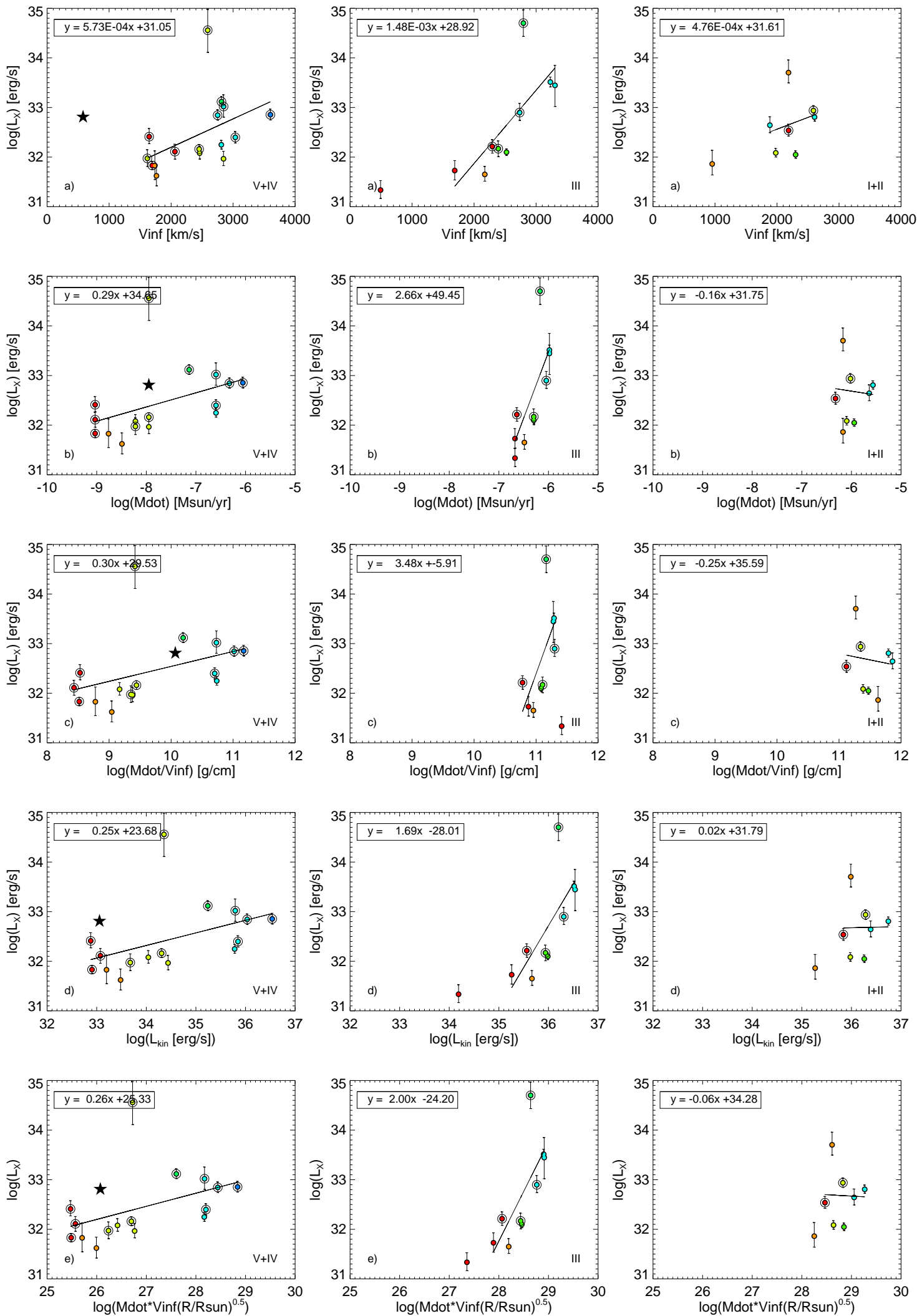

Fig. 12. X-ray luminosity as a function of wind parameters. Only the stars with wind velocities measured from the UV spectra are shown. The mass-loss rates are uncertain within a factor of 2-3 due to systematic errors, i.e., $\Delta \log \dot{M} \approx 0.3-0.5$ (see Sect. 3.4 for a discussion on errors). From left to right columns: results for dwarfs, giants, and supergiants. First row from the top: correlations with wind terminal velocity; second row from the top: dependence on mass-loss rate calculated using the empirical SpT- $\dot{M}$ relations derived in Sect. 3.4; middle row: dependence on wind density; fourth row from the top: dependence on wind kinetic luminosity; bottom row: dependence of X-ray luminosity on modified stellar wind momentum. The color coding of different spectral subtypes is the same as in Fig. 5. Linear fits are shown with a line. 
Table 5. Pearson $(R)$ and Spearman's Rank $(\rho)$ correlation coefficients (see text for descriptions) and fit coefficients for each luminosity class (LC).

\begin{tabular}{|c|c|c|c|c|}
\hline $\mathrm{LC}$ & $R$ & $\rho$ & $a$ & $b$ \\
\hline \multicolumn{5}{|c|}{$\begin{array}{l}\text { Terminal velocity } \\
\log L_{\mathrm{X}}=a+b \times v_{\infty}\end{array}$} \\
\hline All & 0.5 & 0.6 & $30.7 \pm 0.6$ & $(8 \pm 2) \times 10^{-4}$ \\
\hline $\mathrm{V}$ & 0.5 & 0.6 & $31.1 \pm 0.7$ & $(6 \pm 3) \times 10^{-4}$ \\
\hline III & 0.8 & 0.8 & $28.9 \pm 1.4$ & $(10 \pm 5) \times 10^{-4}$ \\
\hline I & 0.2 & 0.2 & $31.6 \pm 2.2$ & $(5 \pm 10) \times 10^{-4}$ \\
\hline \multicolumn{5}{|c|}{ Mass-loss rate } \\
\hline \multicolumn{5}{|c|}{$\log L_{\mathrm{X}}=a+b \times \log \dot{M}$} \\
\hline All & 0.4 & 0.5 & $34.3 \pm 0.8$ & $0.3 \pm 0.1$ \\
\hline $\mathrm{V}$ & 0.4 & 0.6 & $34.7 \pm 1.4$ & $0.3 \pm 0.2$ \\
\hline III & 0.7 & 0.8 & $49.5 \pm 6.8$ & $2.7 \pm 1.1$ \\
\hline I & -0.1 & -0.1 & $31.8 \pm 6.0$ & $-0.2 \pm 1.0$ \\
\hline \multicolumn{5}{|c|}{ Kinetic luminosity } \\
\hline \multicolumn{5}{|c|}{$\log L_{\mathrm{X}}=a+b \times \log L_{\mathrm{kin}}$} \\
\hline All & 0.4 & 0.6 & $23.6 \pm 3.8$ & $0.3 \pm 0.1$ \\
\hline V & 0.4 & 0.6 & $23.7 \pm 5.0$ & $0.3 \pm 0.1$ \\
\hline III & 0.7 & 0.7 & $-28.0 \pm 22.8$ & $1.7 \pm 0.6$ \\
\hline I & 0.0 & 0.3 & $31.8 \pm 32.7$ & $0.0 \pm 0.9$ \\
\hline \multicolumn{5}{|c|}{ Wind density } \\
\hline \multicolumn{5}{|c|}{$\log L_{\mathrm{X}}=a+b \times \log N_{1}$} \\
\hline All & 0.3 & 0.4 & $30.1 \pm 1.3$ & $0.2 \pm 0.1$ \\
\hline $\mathrm{V}$ & 0.4 & 0.6 & $29.5 \pm 1.9$ & $0.3 \pm 0.2$ \\
\hline III & 0.7 & 0.7 & $-5.9 \pm 18.4$ & $3.5 \pm 1.7$ \\
\hline I & -0.1 & -0.2 & $35.6 \pm 11.6$ & $-0.3 \pm 1.0$ \\
\hline \multicolumn{5}{|c|}{ Wind momentum } \\
\hline \multicolumn{5}{|c|}{$\log L_{X}=a+b \times \log D$} \\
\hline All & 0.4 & 0.5 & $25.9 \pm 3.0$ & $0.2 \pm 0.1$ \\
\hline $\mathrm{V}$ & 0.4 & 0.6 & $25.3 \pm 4.2$ & $0.3 \pm 0.2$ \\
\hline III & 0.7 & 0.7 & $-24.2 \pm 21.9$ & $2.0 \pm 0.8$ \\
\hline I & -0.0 & -0.1 & $34.3 \pm 29.0$ & $-0.1 \pm 1.0$ \\
\hline
\end{tabular}

values up to $\log \left(L_{\mathrm{X}} / L_{\mathrm{bol}}\right) \approx-5$. We find that the X-ray to bolometric luminosity ratio depends on the spectral type: $O$ stars with earlier subtypes are X-ray brighter than with later subtypes. We finally find that $\log \left(L_{\mathrm{X}} / L_{\mathrm{bol}}\right)$ is independent of whether the stars are single or in a binary system, a result in agreement with previous studies.

Acknowledgements. We would like to thank the anonymous referee and the editor for their useful comments and suggestions that led to the improvement of the manuscript. The authors are grateful to Dr. W.-R. Hamann for careful reading of the manuscript. We thank Integrated Activities in the High Energy Astrophysics Domain project for support that enabled this work. This work has been financially supported by the Programme National Hautes Energies (PNHE), project 994584 and the Deutsches Zentrum für Luft und Raumfahrt (DLR) grant FKZ 50 OR 1508. LMO acknowledges partial support by the Russian Government Program of Competitive Growth of Kazan Federal University. We thank Integrated Activities in the High Energy Astrophysics Domain project for support that enabled this work. We thank Integrated Activities in the High Energy Astrophysics Domain project for support that enabled this work. This research has made use of data obtained from the 3XMM XMM-Newton serendipitous source catalog compiled by the 10 institutes of the XMM-Newton Survey Science Centre selected by ESA. This research has made use of the SIMBAD database, and the VizieR catalog access tool, operated at CDS, Strasbourg, France.

\section{References}

Abbott, D. C. 1978, ApJ, 225, 893

Antokhin, I. I., Rauw, G., Vreux, J.-M., van der Hucht, K. A., \& Brown, J. C. 2008, A\&A, 477, 593
Bailer-Jones, C. A. L., Rybizki, J., Fouesneau, M., Mantelet, G., \& Andrae, R. 2018, AJ, 158, 58

Berghoefer, T. W., Schmitt, J. H. M. M., Danner, R., \& Cassinelli, J. P. 1997, A\&A, 322, 167

Bohlin, R. C., Savage, B. D., \& Drake, J. F. 1978, ApJ, 224, 132

Bouret, J.-C., Hillier, D. J., Lanz, T., \& Fullerton, A. W. 2012, A\&A, 544, A67 Cantiello, M., \& Braithwaite, J. 2011, A\&A, 534, A140

Cardelli, J. A., Clayton, G. C., \& Mathis, J. S. 1989, ApJ, 345, 245

Castor, J. I., Abbott, D. C., \& Klein, R. I. 1975, ApJ, 195, 157

Chlebowski, T., Harnden, F. R., Jr, \& Sciortino, S. 1989, ApJ, 341, 427

Cutri, R. M., Skrutskie, M. F., van Dyk, S., et al. 2003, VizieR Online Data Catalog : II/246

den Herder, J. W., Brinkman, A. C., Kahn, S. M., et al. 2001, A\&A, 365, L7

Donati, J.-F., Babel, J., Harries, T. J., et al. 2002, MNRAS, 333, 55

Feldmeier, A., Puls, J., \& Pauldrach, A. W. A. 1997, A\&A, 322, 878

Fullerton, A. W., Massa, D. L., \& Prinja, R. K. 2006, ApJ, 637, 1025

Grunhut, J. H., Wade, G. A., Marcolino, W. L. F., et al. 2009, MNRAS, 400, L94

Grunhut, J. H., Wade, G. A., Neiner, C., et al. 2017, MNRAS, 465, 2432

Gudennavar, S. B., Bubbly, S. G., Preethi, K., \& Murthy, J. 2012, ApJS, 199, 8

Hamann, W.-R., \& Gräfener, G. 2003, A\&A, 410, 993

Harnden, F. R., Jr, Branduardi, G., Gorenstein, P., et al. 1979, ApJ, 234, L51

Hillier, D. J., \& Miller, D. L. 1998, ApJ, 496, 407

Hillier, D. J., Kudritzki, R. P., Pauldrach, A. W., et al. 1993, A\&A, 276, 117

Huenemoerder, D. P., Oskinova, L. M., Ignace, R., et al. 2012, ApJ, 756, L34

Ignace, R., Oskinova, L. M., \& Foullon, C. 2000, MNRAS, 318, 214

Indebetouw, R., Mathis, J. S., Babler, B. L., et al. 2005, ApJ, 619, 931

Jansen, F., Lumb, D., Altieri, B., et al. 2001, A\&A, 365, L1

Jenkins, E. B. 2009, ApJ, 700, 1299

Kharchenko, N. V., Piskunov, A. E., Röser, S., Schilbach, E., \& Scholz, R. D. 2005, A\&A, 438, 1163

Kudritzki, R.-P., \& Puls, J. 2000, ARA\&A, 38, 613

Lamers, H. J. G. L. M., \& Cassinelli, J. P. 1999, Introduction to Stellar Winds, 452

Liszt, H. 2014, ApJ, 780, 10

Liu, Q. Z., van Paradijs, J., \& van den Heuvel, E. P. J. 2006, A\&A, 455, 1165

Long, K. S., \& White, R. L. 1980, ApJ, 239, L65

Lucy, L. B., \& White, R. L. 1980, ApJ, 241, 300

Maíz Apellániz, J., Sota, A., Arias, J. I., et al. 2016, ApJS, 224, 4

Martínez-Núñez, S., Kretschmar, P., Bozzo, E., et al. 2017, Space Sci. Rev., 212, 59

Martins, F., \& Plez, B. 2006, A\&A, 457, 637

Martins, F., Schaerer, D., \& Hillier, D. J. 2005a, A\&A, 436, 1049

Martins, F., Schaerer, D., Hillier, D. J., et al. 2005b, A\&A, 441, 735

Massa, D., Oskinova, L., Fullerton, A. W., et al. 2014, MNRAS, 441, 2173

Megier, A., Strobel, A., Galazutdinov, G. A., \& Krełowski, J. 2009, A\&A, 507, 833

Mel'Nik, A. M., \& Dambis, A. K. 2009, MNRAS, 400, 518

Moffat, A. F. J., Corcoran, M. F., Stevens, I. R., et al. 2002, ApJ, 573, 191

Mokiem, M. R., de Koter, A., Vink, J. S., et al. 2007, A\&A, 473, 603

Motch, C., Guillout, P., Haberl, F., et al. 1997, A\&A, 318, 111

Muijres, L. E., Vink, J. S., de Koter, A., Müller, P. E., \& Langer, N. 2012, A\&A, 537, A37

Nazé, Y. 2009, A\&A, 506, 1055

Nazé, Y., Broos, P. S., Oskinova, L., et al. 2011, ApJS, 194, 7

Nazé, Y., Oskinova, L. M., \& Gosset, E. 2013, ApJ, 763, 143

Nazé, Y., Petit, V., Rinbrand, M., et al. 2014, ApJS, 215, 10

Ochsenbein, F., Bauer, P., \& Marcout, J. 2000, A\&AS, 143, 23

Oskinova, L. M. 2005, MNRAS, 361, 679

Oskinova, L. M., Clarke, D., \& Pollock, A. M. T. 2001, A\&A, 378, L21

Oskinova, L. M., Feldmeier, A., \& Hamann, W.-R. 2006, MNRAS, 372, 313

Oskinova, L. M., Hamann, W.-R., \& Feldmeier, A. 2007, A\&A, 476, 1331

Oskinova, L. M., Hamann, W.-R., Cassinelli, J. P., Brown, J. C., \& Todt, H. 2011 , Astron. Nachr., 332, 988

Oskinova, L. M., Kubátová, B., \& Hamann, W.-R. 2016, J. Quant. Spectr. Rad. Transf., 183, 100

Owocki, S. P., Castor, J. I., \& Rybicki, G. B. 1988, ApJ, 335, 914

Owocki, S. P., Sundqvist, J. O., Cohen, D. H., \& Gayley, K. G. 2013, MNRAS, 429, 3379

Pallavicini, R., Golub, L., Rosner, R., et al. 1981, ApJ, 248, 279

Pandey, A. K., Sandhu, T. S., Sagar, R., \& Battinelli, P. 2010, MNRAS, 403, 1491

Predehl, P., \& Schmitt, J. H. M. M. 1995, A\&A, 293, 889

Prinja, R. K., Barlow, M. J., \& Howarth, I. D. 1990, ApJ, 361, 607

Puls, J., Urbaneja, M. A., Venero, R., et al. 2005, A\&A, 435, 669

Ramiaramanantsoa, T., Moffat, A. F. J., Chené, A.-N., et al. 2014, MNRAS, 441, 910

Reed, B. C. 2003, AJ, 125, 2531 
Repolust, T., Puls, J., \& Herrero, A. 2004, A\&A, 415, 349

Rosen, S. R., Webb, N. A., Watson, M. G., et al. 2016, A\&A, 590, A1

Sana, H., Rauw, G., Nazé, Y., Gosset, E., \& Vreux, J.-M. 2006, MNRAS, 372, 661

Schmitt, J. H. M. M., Golub, L., Harnden, F. R., Jr, et al. 1985, ApJ, 290, 307

Schöller, M., Hubrig, S., Fossati, L., et al. 2017, A\&A, 599, A66

Sciortino, S., Vaiana, G. S., Harnden, F. R., Jr, et al. 1990, ApJ, 361, 621

Shenar, T., Oskinova, L., Hamann, W.-R., et al. 2015, ApJ, 809, 135

Shenar, T., Oskinova, L. M., Järvinen, S. P., et al. 2017, A\&A, 606, A91

Skiff, B. A. 2014, VizieR Online Data Catalog: B/mk

Smith, R. K., Brickhouse, N. S., Liedahl, D. A., \& Raymond, J. C. 2001, ApJ, 556, L91

Sota, A., Maíz Apellániz, J., Walborn, N. R., et al. 2011, ApJS, 193, 24

Sota, A., Maíz Apellániz, J., Morrell, N. I., et al. 2014, ApJS, 211, 10
Strüder, L., Briel, U., Dennerl, K., et al. 2001, A\&A, 365, L18

Sundqvist, J. O., Puls, J., Feldmeier, A., \& Owocki, S. P. 2011, A\&A, 528, A64

Šurlan, B., Hamann, W.-R., Kubát, J., Oskinova, L. M., \& Feldmeier, A. 2012, A\&A, 541, A37

Šurlan, B., Hamann, W.-R., Aret, A., et al. 2013, A\&A, 559, A130

Turner, M. J. L., Abbey, A., Arnaud, M., et al. 2001, A\&A, 365, L27 Urpin, V. 2017, MNRAS, 472, L5

Vink, J. S., de Koter, A., \& Lamers, H. J. G. L. M. 2000, A\&A, 362, 295

Voges, W., Aschenbach, B., Boller, T., et al. 1999, A\&A, 349, 389

Voges, W., Aschenbach, B., Boller, T., et al. 2000, IAU Circ., 7432

Waldron, W. L., \& Cassinelli, J. P. 2007, ApJ, 668, 456

Watson, M. G., Schröder, A. C., Fyfe, D., et al. 2009, A\&A, 493, 339

Wenger, M., Ochsenbein, F., Egret, D., et al. 2000, A\&AS, 143, 9

Wolk, S. J., Spitzbart, B. D., Bourke, T. L., \& Alves, J. 2006, AJ, 132, 1100 


\section{Appendix A: Soft and hard X-ray to bolometric luminosities}

For completeness we investigated the $\log \left(f_{\mathrm{X}} / f_{\text {bol }}\right)$ relation in two different energy bands: in soft X-rays $(0.5-2.5 \mathrm{keV})$ and in hard X-rays $(2.5-12 \mathrm{keV})$. The correlation between $\log \left(f_{\mathrm{X}}\right)$ and $\log \left(f_{\text {bol }}\right)$ is strong in the soft energy band and moderate in the hard energy band, as shown in Fig. A.1 (we note the different scale in the ordinate axis).
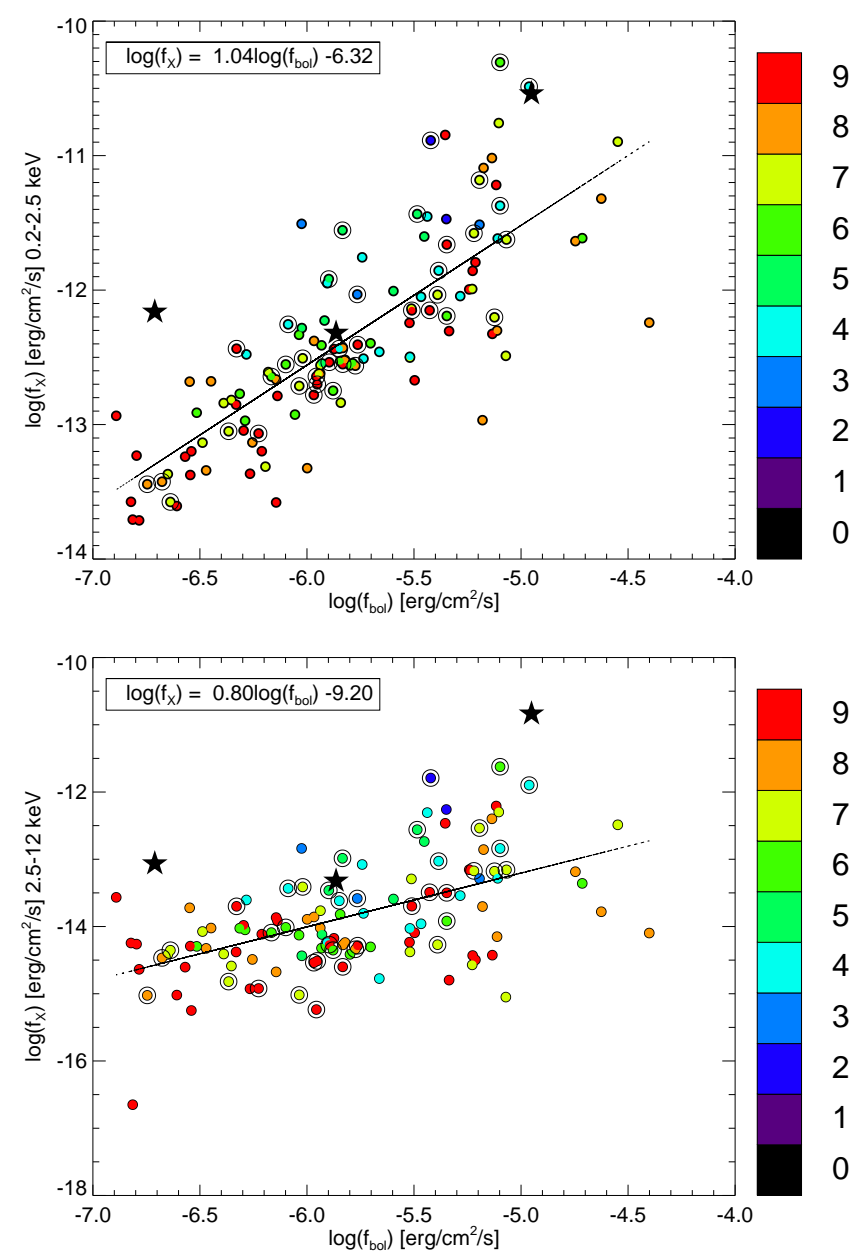

Fig. A.1. $\log \left(f_{\mathrm{X}} / f_{\text {bol }}\right)$ relation for O stars of all luminosity classes and as a function of the spectral subtype (color coded). Known spectroscopic binaries are highlighted with a ring around the solid circle and magnetic $\mathrm{O}$ stars are shown with an asterisk. In the top panel the $\log \left(f_{\mathrm{X}} / f_{\mathrm{bol}}\right)$ relation in the soft band $(0.5-2.5 \mathrm{keV})$ is shown, while in the bottom panel the same is shown in hard X-ray band $(2.5-12 \mathrm{keV})$.

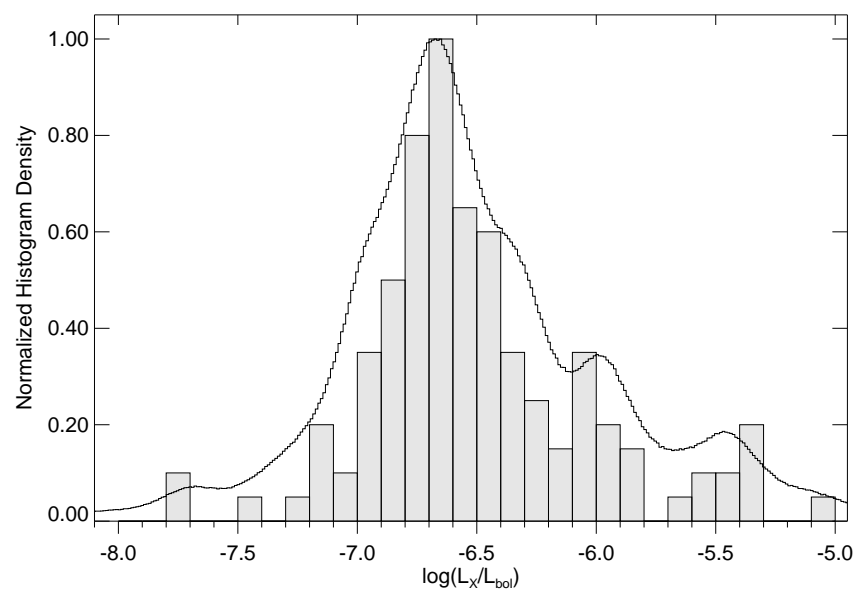

Fig. B.1. $\log \left(L_{\mathrm{X}} / L_{\mathrm{bol}}\right)$ distribution of our sample stars is shown as a gray histogram and Monte Carlo simulated distribution (see text for details) is shown as a black line.

\section{Appendix B: Errors in the $\log \left(L_{x} / L_{\text {bol }}\right)$ distribution}

To investigate the impact of the estimated errors on the $\log \left(L_{\mathrm{X}} / L_{\text {bol }}\right)$ distribution we performed a Monte Carlo simulation. For each star we assigned 10000 random fluxes normally distributed with mean equal to the measured value and $\sigma$ equal to error. Since for $\log \left(L_{\mathrm{X}} / L_{\mathrm{bol}}\right)$ we determined upper and lower limits we took as error the maximum difference between these limits and the measured value. We then calculated the normalized histogram of the distribution for these random values. The result is shown in Fig. B.1 together with the measured distribution. The overall shapes of both distributions are similar, presenting a peak at around the same value and right-skewed.

\section{Appendix C: Bolometric luminosity as a function of wind parameters}

The bolometric luminosity is shown as a function of wind parameters in Figs. C.1 and C.2, as a function of luminosity class (dwarfs and subdwarfs in the left panels, giants in the middle panels, and supergiants in the right panels). While dwarf and giant stars stars show a moderate correlation with the terminal velocity, supergiant stars do not. Wind kinetic luminosity, momentum, and density are dependent on $v_{\infty}$ and $\dot{M}$. Therefore, the correlations with $L_{\mathrm{bol}}$ are reflecting a correlation with these two fundamental parameters. While in Fig. C.1 luminosities have been calculated based on the parallactic distances (Bailer-Jones et al. 2018), in Fig. C.2 luminosities are determined using the tabulated values by Martins et al. (2005a) for different spectral types and luminosity classes. 
A. Nebot Gómez-Morán and L. M. Oskinova: X-ray catalog of spectroscopically identified Galactic O stars
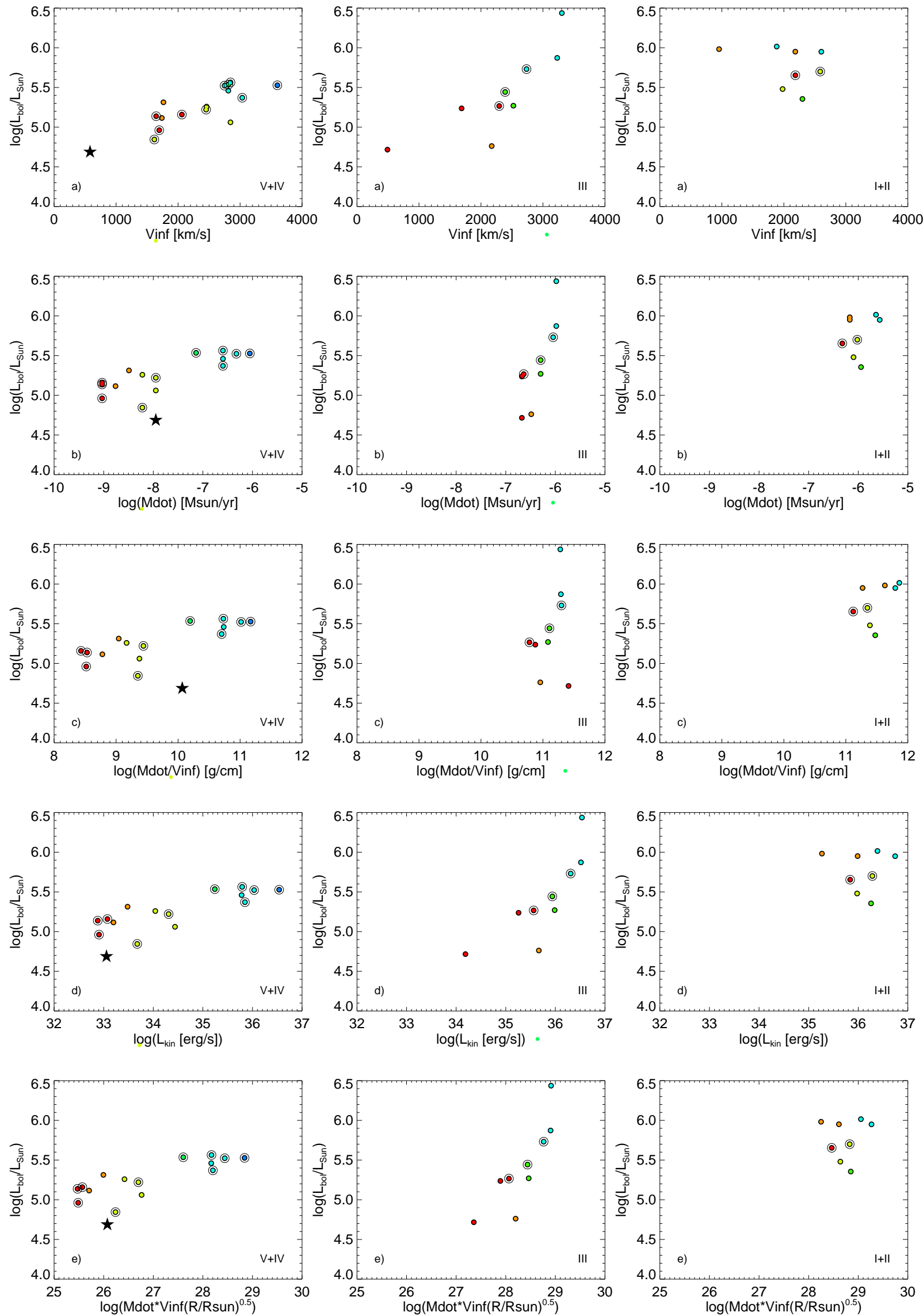

Fig. C.1. Bolometric luminosity against terminal wind velocity (panel a), mass-loss rate calculated using the empirical SpT- $\dot{M}$ relations derived in Sect. 3.4 (panel b), wind density (panel c), kinetic wind luminosity (panel $d$ ) and modified stellar wind momentum (panel $e$ ) for different luminosity classes in each panel. Symbols have been color coded according to spectral type. 
A\&A 620, A89 (2018)
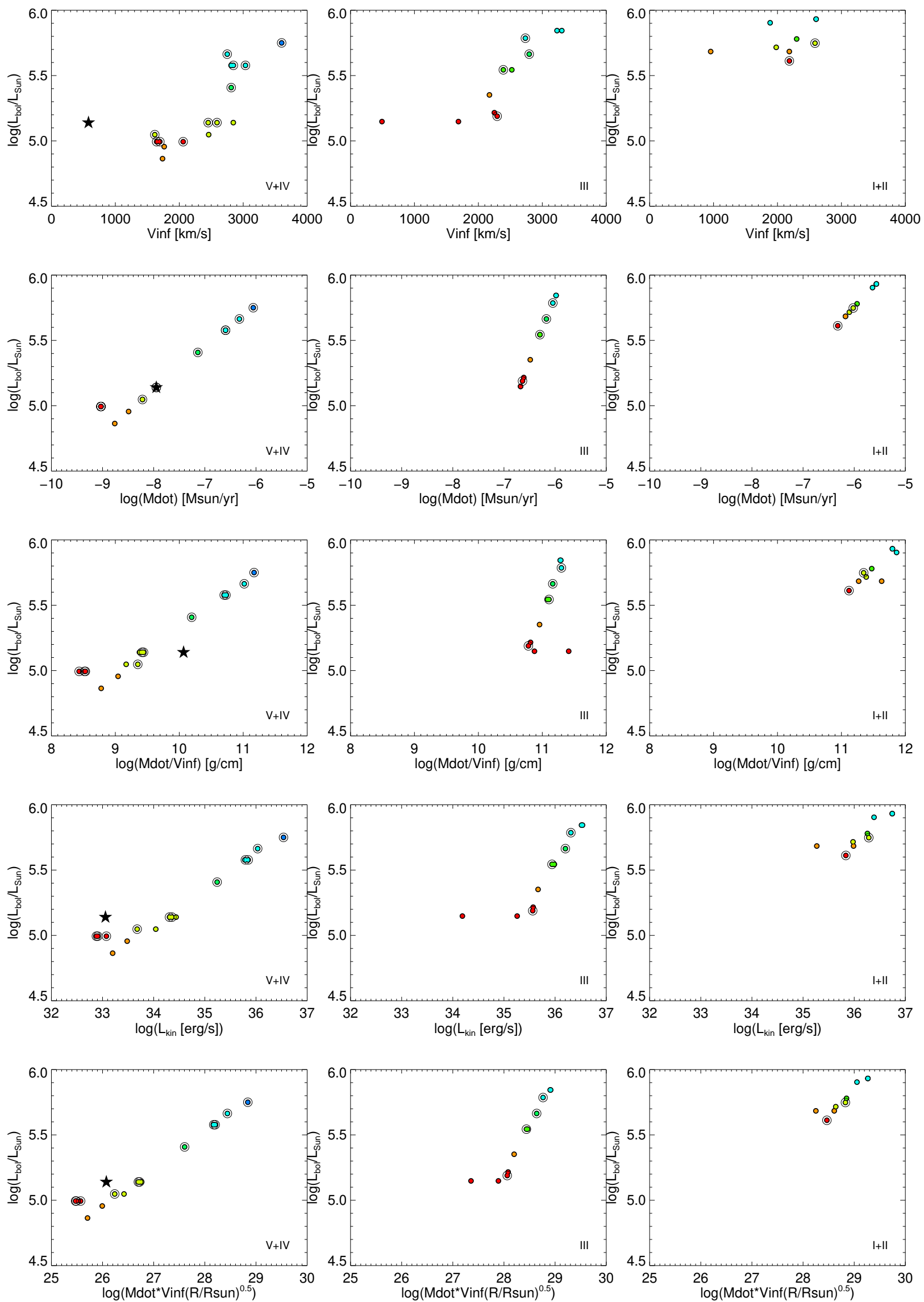

Fig. C.2. Bolometric luminosity against terminal wind velocity (panel a), mass-loss rate calculated using the empirical SpT- $\dot{M}$ relations derived in Sect. 3.4 (panel b), wind density (panel c), kinetic wind luminosity (panel $d$ ) and modified stellar wind momentum (panel $e$ ) for different luminosity classes in each panel. Symbols have been color coded according to spectral type. 